\title{
AVIFAUNAL COMPOSITION OF VARIOUS MICROHABITATS OF SOUTHERN NAGAUR (PARBATSAR, KUCHAMAN, NAWA AND MAKRANA), RAJASTHAN
}

\author{
Rounak Choudhary ${ }^{1}$, Praveen Mathur ${ }^{1}$, Vivek Sharma *1凶 \\ ${ }^{* 1}$ Department of Environmental Sciences, M.D.S. University, Ajmer, India
}

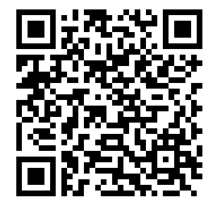

DOI: https://doi.org/10.29121/granthaalayah.v8.i11.2020.2318

Article Type: Research Article

Article Citation: Rounak Choudhary, Praveen Mathur, and Vivek Sharma. (2020). AVIFAUNAL COMPOSITION OF VARIOUS MICROHABITATS OF SOUTHERN NAGAUR (PARBATSAR, KUCHAMAN, NAWA AND MAKRANA), RAJASTHAN. International Journal of Research GRANTHAALAYAH, 8(11), 112-128. https://doi.org/10.29121/granthaa layah.v8.i11.2020.2318

Received Date: 01 November 2020

Accepted Date: 30 November 2020

Keywords:

Avifauna

Microhabitats

Nagaur

Parbatsar

Nawa

Kuchaman

Makrana

Feeding Guilds

\section{ABSTRACT}

Present study comprises a comprehensive dataset about the Avifaunal diversity of one of the climatic transition zone i.e., Southern Nagur, Rajasthan. The Study was conducted under the classified six different microhabitats i.e., Agricultural Field Plains, Sambhar Lake and Other Waterbodies, Aravalli Hill Patches, Urban Settlements, Scrub Thorn Forest and Open Grasslands \& Wastelands. The key aspect of present study is to provide well structured and extensive information on the avian diversity, their distribution and their habitat association at the study area. A total of 191 Avifaunal species belonging to 21 Orders and 61 Families were recorded from study area. A total of 7 Feeding Guilds were observed in the study area viz., Carnivores, Frugivores, Granivores, Herbivores, Insectivores, Nectivores and Omnivored. The Migratory status and IUCN status of the observed species was also assessed. Jaccard and Sorenson index's was highest for the Agricultural and Grassland Microhabitats.

\section{INTRODUCTION}

The state of Rajasthan can be divided into two major climatic zones i.e. Arid and Semiarid, by the Aravalli Mountain ranges. The districts lying near Aravalli mountain ranges act as a transition zone between such two climatic zones. With variety of microhabitats in the state of Rajasthan along with two Ramsar Sites i.e., are Keoladeo National Park and Sambhar Lake (Islam and Rahmani, 2004). Rajasthan is rich in various floral and faunal components including the avifaunal diversity with estimation of about 500 species of birds (BNHS, 2011b).

Birds are one of the best indicators of environmental quality, health and they easily depicted significant variations against changes in the microhabitat quality in both the terms spatial as well as temporal. Birds also exhibit the relationship with the structure and characteristics of the microhabitat (Cody, 1978). The population and

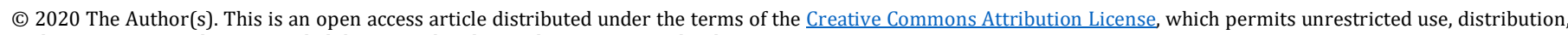
and reproduction in any medium, provided the original author and source are credited. 
community structure of bird is directly affected by the food availability, nest site availability, human exposure and presence of other threat (Wiens, 1989). Studying the bird population is useful to monitor the long term changes in habitat quality and responses of birds to both natural and anthropogenic induced environmental changes (Wiens, 1989).

As, this is well known fact, that the transition zones exhibited greater amount of diversity as they shared nearly all characteristics of both the nearby microhabitats. Inspite of such importance still many transition zones including the Nagaur district are not evaluated properly for their faunal diversity including the avifaunal compositions. The present study is an approach to fulfill such gap as it comprises the avifaunal diversity of southern region of Naguar district in classified six different microhabitats i.e., Agricultural Field Plains, Sambhar Lake and Other Waterbodies, Aravalli Hill Patches, Urban Settlements, Scrub Thorn Forest and Open Grasslands \& Wastelands. The main purpose of this study is to provide well structured and extensive information on the avian diversity, distribution and habitat association of avifauna of southern region of Nagaur, administratively comes under the Parbatsar, Nawa, Makrana and Kuchaman towns of Nagaur District.

\subsection{OBJECTIVES OF THE STUDY}

The objective of present study is to prepare an avifaunal inventory of the study area by observing all six microhabitats and to identify the feeding guild (Simberloff and Dayan 1991), IUCN Status (IUCN, 2020) and Migration Pattern (Able, 1995) of the documented bird species of the study area.

\section{MATERIALS AND METHODS}

\subsection{PROFILE OF STUDY AREA}

The study was carried out in six microhabitats at southern region of Nagaur district, Rajasthan. The study area located between $26^{\circ} 54^{\prime} 20.1^{\prime \prime} \mathrm{N} 74^{\circ} 46^{\prime} 31.5^{\prime \prime} \mathrm{E}$ at transition zone between arid and semiarid climatic zones of Rajasthan.

Four seasons are recognized at the study area based on rainfall i.e., Summer (April-June), Pre-monsoon (JulySeptember), Monsoon (October-December) and Post-monsoon (January-March). The December to January is the coolest time period and May to June is the warmest period at the study area. The study area is well dominated by Agricultural Lands followed by Scrub Thorn Forest and Wasteland. The western parts of the study area are covered by Aravalli Mountain Ranges. Soils are relatively fertile and support all agricultural activity in the study area. Four types of soils have been reported from the study area viz., Clay, Clay Loam, Sandy Loam and Sandy Soil. The area lies under the transition zone of arid and semiarid climate and is represented by chiefly Dry mixed deciduous thorn forest along with some bushy plants Anogeissus pendula and Capparis decidua. The plainer parts of the study area is dominated by Acacia nilotica, Acacia senegal, Salvadora persica etc. The nearby of seasonal rivers and streams the soil is of sandy with good amount of moisture and mainly populated by the Dalbergia sissoo as the common tree, while other important species are Azadirachta indica, Prosopis cineraria and Prosopis juliflora. The degraded areas at the catchment are dominated by an invasive plant Prosopis juliflora. Due to high saline condition the other species does not survive easily at the study area but the growth of Prosopis juliflora is in abundance.

Agro-Ecosystems or Agricultural area covers the largest portion of study area. The area is mostly cultivated during the monsoon season. Some of the important crops like Pearl millet, Cluster bean, Sesame, Green gram, Moth bean are the main seasonal crops. However, in more fertile soil groundnut, wheat, cumin, cotton, mustard and gram are also grown on fairly large scale. 

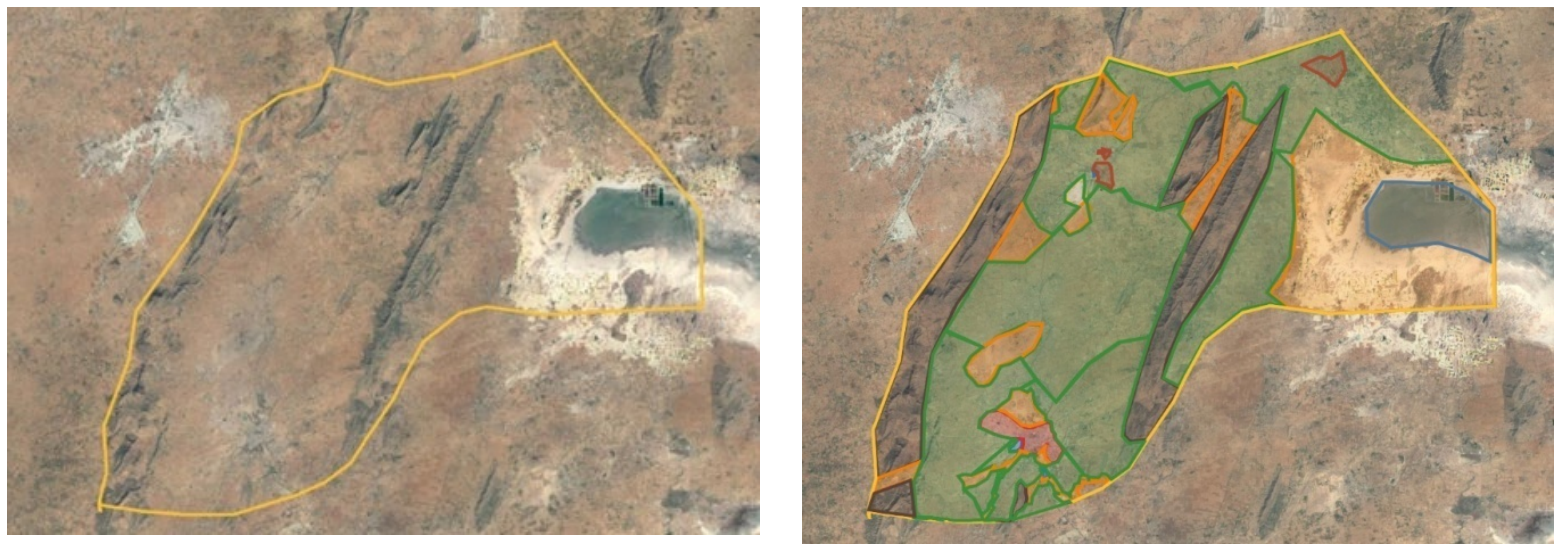

Figure 1: (A) Satellite map of Study Area, (B) With highlighted microhabitats

\subsection{DATA COLLECTION}

Regular field surveys were carried out from August 2019 to August 2020 resulting in a total 52 surveys in six microhabitats: 20 in summer, 20 in winter and 12 in monsoon. The length of transect remained constant in all the surveys that is $1000 \mathrm{~m}(1 \mathrm{KM})$. The time remained constant in all microhabitat, morning surveys was carried out from 6:00 Am to 9:00 Am and evening surveys was carried out from 4:00 Pm to 7:00 Pm. Olympus Binocular 10*50X was used for on field observations and Canon- Eos 1300D camera was used for photography. For identification of birds "Book of Indian Birds" by Salim ali (Ali, 1992) and "A field guide on the birds of the Indian subcontinent" Grimmett (Grimmett et al., 1998) was referred. Checklist was prepared using various methods like Adhoc, Area Search Method, Point Count Method, Rolling Bird Survey Method and Call Based Identification Method (Urfi et al., 2005).

\subsection{DATA ANALYSIS}

\section{Relative Diversity Index (RDi)}

The relative diversity (RDi) of families was calculated (Torre-Cuadros et al., 2007).

$$
R D i=\frac{\text { Number of bird species in a family }}{\text { Total number of bird species }} \times 100
$$

\section{Sorenson Index}

To assess the association of species between two study sites, Sorensen's index of similarity (Sorenson, 1948) was calculated.

$$
C_{s}=\frac{2 j}{(a+b)}
$$

Where $\mathrm{j}=$ number of species common to both sites; $\mathrm{a}=$ number of species at site $\mathrm{A} ; \mathrm{b}=$ number of species at site

\section{Jaccard Index}

To assess the association of species between two study sites, Jaccard index of similarity was calculated.

$$
C_{j}=\frac{j}{(a+b-j)}
$$


Avifaunal Composition of Various Microhabitats of Southern Nagaur (Parbatsar, Kuchaman, Nawa and Makrana), Rajasthan

B.

Where $\mathrm{j}=$ number of species common to both sites; $\mathrm{a}=$ number of species at site $\mathrm{A} ; \mathrm{b}=$ number of species at site

\section{RESULTS AND DISCUSSION}

A total of 191 Avifaunal species were recorded from study area (Table 1). Total 21 Orders were recorded from the study area (Table 2). Order Passeriformes dominated the study area with 71 Bird species followed by order Charadriiformes (28), Anseriformes (14), Pelecaniformes (13) and Accipitriformes (12). A total of 61 Families were recorded from study area (Table 3). Family Anatidae dominated the study area with 14 species followed by Muscicapidae (12), Accipitridae (11) and Scolopacide (11) with relative diversity value of 7.33\%, 6.28\%, 5.76\% and $5.76 \%$ respectively. A total of 7 Feeding Guilds were observed in the study area viz., Carnivores, Frugivores, Granivores, Herbivores, Insectivores, Nectivores and Omnivored based on the food preferences of the Avifauna (Table 4 \& Fig. 2).

Most Birds in study area preferred Insectivore feeding guild as of 77 species of total 191 are insectivore followed by Carnivores (45), Granivores (24), Omnivores (23), Herbivores (11), Frugivores (10) and only one species of Nectivores. 119 out of total 191 species recorded in study area were identified as residential species of the area, 72 species were identified as Migratory species out of which 5 as Summer Migratory and 67 as Winter Migratory (Table 5 \& Fig. 3).

In recorded 191 species, a total of 4 IUCN Red List Categories out of 7 was observed in the study area. 178 Species out of 191 were identified as Least Concerned Species. 8 Species were identified as Near Threatened species which are Ferruginous pochard, Great thick knee, River tern, Black tailed godwit, Painted Stork, Oriental White Ibis, Lesser Flamingo, Alexendrine Parakeet. 3 Species i.e. Common Pochard, Woolly Necked Stork, Southern Grey Shrike were identified as Vulnerable, And 2 Species viz., Egyptian Vulture and Steppe Eagle as Endangered (Table 6 \& Fig. 4).

During the entire period of study the maximum species was observed from the Water bodies microhabitat (95 species) followed by Scrub forest microhabitats (89). In contrast the minimum species represented by Urban settlements microhabitat (38 species). The higher amount of species diversity indicated the better quality and resource availability at that particular microhabitat.

Jaccard and Sorenson index's increasing values indicates the similarity between two microhabitats. The Agricultural and Grassland Microhabitats have highest values (Jaccard= 0.559, Sorenson= 0.717) are much similar to each other.

Table 1: Order, Family, Common Name, Scientific Name, Feeding Guild, Migratory Status and IUCN Status of Observed Species

\begin{tabular}{|c|c|c|c|c|c|c|c|}
\hline $\begin{array}{l}\text { S. } \\
\text { N } \\
\text { O }\end{array}$ & Order & Family & Common name & Scientific name & $\begin{array}{c}\text { Feeding } \\
\text { Guild }\end{array}$ & $\begin{array}{c}\text { Migratory } \\
\text { Status }\end{array}$ & $\begin{array}{l}\text { IUCN } \\
\text { Status }\end{array}$ \\
\hline 1 & $\begin{array}{l}\text { Accipitrifor } \\
\text { mes }\end{array}$ & $\begin{array}{c}\text { Accipitrida } \\
\mathrm{e}\end{array}$ & Black winged kite & Elanus axillaris & $\begin{array}{l}\text { Carnivo } \\
\text { rous }\end{array}$ & $\mathrm{R}$ & $\begin{array}{c}\text { Least } \\
\text { concern }\end{array}$ \\
\hline 2 & $\begin{array}{l}\text { Accipitrifor } \\
\text { mes }\end{array}$ & $\begin{array}{c}\text { Accipitrida } \\
\text { e }\end{array}$ & Black kite & Milvus migrans & $\begin{array}{c}\text { Carnivo } \\
\text { rous }\end{array}$ & $\mathrm{R}$ & $\begin{array}{c}\text { Least } \\
\text { concern }\end{array}$ \\
\hline 3 & $\begin{array}{l}\text { Accipitrifor } \\
\text { mes }\end{array}$ & $\begin{array}{c}\text { Accipitrida } \\
\text { e }\end{array}$ & Shikra & Accipiter badius & $\begin{array}{l}\text { Carnivo } \\
\text { rous }\end{array}$ & $\mathrm{R}$ & $\begin{array}{c}\text { Least } \\
\text { concern }\end{array}$ \\
\hline 4 & $\begin{array}{l}\text { Accipitrifor } \\
\text { mes }\end{array}$ & $\begin{array}{c}\text { Accipitrida } \\
\text { e }\end{array}$ & $\begin{array}{c}\text { Oriental honey } \\
\text { buzzard }\end{array}$ & $\begin{array}{c}\text { Pernis } \\
\text { ptilorhynchus }\end{array}$ & $\begin{array}{l}\text { Carnivo } \\
\text { rous }\end{array}$ & $\mathrm{R}$ & $\begin{array}{c}\text { Least } \\
\text { concern }\end{array}$ \\
\hline 5 & $\begin{array}{l}\text { Accipitrifor } \\
\text { mes }\end{array}$ & $\begin{array}{c}\text { Accipitrida } \\
\text { e }\end{array}$ & $\begin{array}{l}\text { White eyed } \\
\text { buzzard }\end{array}$ & Butastur teesa & $\begin{array}{l}\text { Carnivo } \\
\text { rous }\end{array}$ & $\mathrm{R}$ & $\begin{array}{c}\text { Least } \\
\text { concern }\end{array}$ \\
\hline 6 & $\begin{array}{l}\text { Accipitrifor } \\
\text { mes }\end{array}$ & $\begin{array}{c}\text { Accipitrida } \\
\text { e }\end{array}$ & $\begin{array}{c}\text { Eurasian Marsh } \\
\text { harrier }\end{array}$ & Ciircus aeruginosus & $\begin{array}{l}\text { Carnivo } \\
\text { rous }\end{array}$ & W & $\begin{array}{c}\text { Least } \\
\text { concern }\end{array}$ \\
\hline 7 & $\begin{array}{l}\text { Accipitrifor } \\
\text { mes }\end{array}$ & $\begin{array}{c}\text { Accipitrida } \\
\text { e }\end{array}$ & Egyptian vulture & $\begin{array}{c}\text { Neophron } \\
\text { percnopterus }\end{array}$ & $\begin{array}{c}\text { Carnivo } \\
\text { rous }\end{array}$ & $\mathrm{R}$ & $\begin{array}{c}\text { Endagere } \\
\mathrm{d}\end{array}$ \\
\hline
\end{tabular}


Rounak Choudhary, Praveen Mathur, and Vivek Sharma

\begin{tabular}{|c|c|c|c|c|c|c|c|}
\hline 8 & $\begin{array}{l}\text { Accipitrifor } \\
\text { mes }\end{array}$ & $\begin{array}{l}\text { Accipitrida } \\
\text { e }\end{array}$ & Montagu's harrier & Circus pygargus & $\begin{array}{l}\text { Carnivo } \\
\text { rous }\end{array}$ & $\mathrm{W}$ & $\begin{array}{l}\text { Least } \\
\text { concern }\end{array}$ \\
\hline 9 & $\begin{array}{l}\text { Accipitrifor } \\
\text { mes }\end{array}$ & $\begin{array}{c}\text { Accipitrida } \\
\text { e }\end{array}$ & Long leg buzzard & Buteo rufinus & $\begin{array}{l}\text { Carnivo } \\
\text { rous }\end{array}$ & $\mathrm{W}$ & $\begin{array}{l}\text { Least } \\
\text { concern }\end{array}$ \\
\hline 10 & $\begin{array}{l}\text { Accipitrifor } \\
\text { mes }\end{array}$ & $\begin{array}{c}\text { Accipitrida } \\
\mathrm{e}\end{array}$ & Steppe eagle & Aquila nipalensis & $\begin{array}{l}\text { Carnivo } \\
\text { rous }\end{array}$ & $\mathrm{W}$ & $\begin{array}{c}\text { Endagere } \\
\mathrm{d}\end{array}$ \\
\hline 11 & $\begin{array}{l}\text { Accipitrifor } \\
\text { mes }\end{array}$ & $\begin{array}{c}\text { Accipitrida } \\
\text { e }\end{array}$ & Common buzzard & Buteo buteo & $\begin{array}{l}\text { Carnivo } \\
\text { rous }\end{array}$ & $\mathrm{W}$ & $\begin{array}{c}\text { Least } \\
\text { concern }\end{array}$ \\
\hline 12 & $\begin{array}{l}\text { Accipitrifor } \\
\text { mes }\end{array}$ & $\begin{array}{l}\text { Pandionida } \\
\text { e }\end{array}$ & Osprey & Pandion haliaetus & $\begin{array}{l}\text { Carnivo } \\
\text { rous }\end{array}$ & $\mathrm{R}$ & $\begin{array}{l}\text { Least } \\
\text { concern }\end{array}$ \\
\hline 13 & $\begin{array}{c}\text { Anseriforme } \\
\text { s }\end{array}$ & Anatidae & Bar headed goose & Anser indicus & $\begin{array}{l}\text { Omnivo } \\
\text { rous }\end{array}$ & $\mathrm{R}$ & $\begin{array}{c}\text { Least } \\
\text { concern }\end{array}$ \\
\hline 14 & $\begin{array}{c}\text { Anseriforme } \\
\mathrm{s} \\
\end{array}$ & Anatidae & Ruddy Shelduck & $\begin{array}{c}\text { Tadorana } \\
\text { ferruginea }\end{array}$ & $\begin{array}{l}\text { Omnivo } \\
\text { rous }\end{array}$ & $\mathrm{W}$ & $\begin{array}{c}\text { Least } \\
\text { concern }\end{array}$ \\
\hline 15 & $\begin{array}{c}\text { Anseriforme } \\
\mathrm{s}\end{array}$ & Anatidae & Common pochard & Aythya ferina & $\begin{array}{l}\text { Omnivo } \\
\text { rous }\end{array}$ & $\mathrm{W}$ & $\begin{array}{c}\text { Vulnerabl } \\
\text { e }\end{array}$ \\
\hline 16 & $\begin{array}{c}\text { Anseriforme } \\
\text { s }\end{array}$ & Anatidae & $\begin{array}{c}\text { Ferrogenous } \\
\text { pochard }\end{array}$ & Aythya nyroca & $\begin{array}{l}\text { Omnivo } \\
\text { rous }\end{array}$ & $\mathrm{W}$ & $\begin{array}{c}\text { Near } \\
\text { Threatene } \\
\text { d }\end{array}$ \\
\hline 17 & $\begin{array}{c}\text { Anseriforme } \\
\mathrm{s}\end{array}$ & Anatidae & Graylag goose & Anser anser & $\begin{array}{l}\text { Herbivo } \\
\text { rous }\end{array}$ & $\mathrm{W}$ & $\begin{array}{c}\text { Least } \\
\text { concern }\end{array}$ \\
\hline 18 & $\begin{array}{c}\text { Anseriforme } \\
\mathrm{s}\end{array}$ & Anatidae & Knob billed duck & $\begin{array}{l}\text { Sarkidiornis } \\
\text { melanotos }\end{array}$ & $\begin{array}{l}\text { Herbivo } \\
\text { rous }\end{array}$ & $\mathrm{R}$ & $\begin{array}{c}\text { Least } \\
\text { concern }\end{array}$ \\
\hline 19 & $\begin{array}{l}\text { Anseriforme } \\
\mathrm{s}\end{array}$ & Anatidae & $\begin{array}{c}\text { Lesser whistling } \\
\text { duck }\end{array}$ & $\begin{array}{c}\text { Dendrocygna } \\
\text { javanica }\end{array}$ & $\begin{array}{l}\text { Herbivo } \\
\text { rous }\end{array}$ & $\mathrm{R}$ & $\begin{array}{c}\text { Least } \\
\text { concern }\end{array}$ \\
\hline 20 & $\begin{array}{l}\text { Anseriforme } \\
\text { s }\end{array}$ & Anatidae & Northern Pintail & Anas acuta & $\begin{array}{l}\text { Herbivo } \\
\text { rous }\end{array}$ & $\mathrm{W}$ & $\begin{array}{c}\text { Least } \\
\text { concern }\end{array}$ \\
\hline 21 & $\begin{array}{l}\text { Anseriforme } \\
\text { s }\end{array}$ & Anatidae & Common teal & Anas crecca & $\begin{array}{l}\text { Herbivo } \\
\text { rous }\end{array}$ & $\mathrm{W}$ & $\begin{array}{l}\text { Least } \\
\text { concern }\end{array}$ \\
\hline 22 & $\begin{array}{l}\text { Anseriforme } \\
\mathrm{s}\end{array}$ & Anatidae & Spot billed duck & $\begin{array}{c}\text { Anas } \\
\text { poecilorhyncha }\end{array}$ & $\begin{array}{l}\text { Herbivo } \\
\text { rous }\end{array}$ & $\mathrm{R}$ & $\begin{array}{c}\text { Least } \\
\text { concern }\end{array}$ \\
\hline 23 & $\begin{array}{c}\text { Anseriforme } \\
\mathrm{s} \\
\end{array}$ & Anatidae & Mallard & Anas platyrhynchos & $\begin{array}{l}\text { Herbivo } \\
\text { rous }\end{array}$ & $\mathrm{W}$ & $\begin{array}{c}\text { Least } \\
\text { concern }\end{array}$ \\
\hline 24 & $\begin{array}{c}\text { Anseriforme } \\
\mathrm{s} \\
\end{array}$ & Anatidae & Gadwall & Anas strepera & $\begin{array}{l}\text { Herbivo } \\
\text { rous }\end{array}$ & $\mathrm{W}$ & $\begin{array}{c}\text { Least } \\
\text { concern }\end{array}$ \\
\hline 25 & $\begin{array}{l}\text { Anseriforme } \\
\mathrm{s}\end{array}$ & Anatidae & Garganey & Anas querquedula & $\begin{array}{l}\text { Herbivo } \\
\text { rous }\end{array}$ & $\mathrm{W}$ & $\begin{array}{c}\text { Least } \\
\text { concern }\end{array}$ \\
\hline 26 & $\begin{array}{l}\text { Anseriforme } \\
\mathrm{S}\end{array}$ & Anatidae & Northern shoveler & Anas clypeata & $\begin{array}{c}\text { Carnivo } \\
\text { rous }\end{array}$ & $\mathrm{W}$ & $\begin{array}{c}\text { Least } \\
\text { concern }\end{array}$ \\
\hline 27 & Apodiformes & Apodidae & House swift & Apus affinis & $\begin{array}{l}\text { Insectiv } \\
\text { orous }\end{array}$ & $\mathrm{R}$ & $\begin{array}{c}\text { Least } \\
\text { concern }\end{array}$ \\
\hline 28 & $\begin{array}{c}\text { Bucerotifor } \\
\text { mes }\end{array}$ & Upupidae & Common hoopoe & Uрира ерорs & $\begin{array}{l}\text { Insectiv } \\
\text { orous }\end{array}$ & $\mathrm{R}$ & $\begin{array}{c}\text { Least } \\
\text { concern }\end{array}$ \\
\hline 29 & $\begin{array}{c}\text { Bucerotifor } \\
\text { mes }\end{array}$ & $\begin{array}{c}\text { Bucerotida } \\
\text { e }\end{array}$ & $\begin{array}{c}\text { Indian grey } \\
\text { hornbill }\end{array}$ & Ocyceros birostris & $\begin{array}{l}\text { Frugivo } \\
\text { rous }\end{array}$ & $\mathrm{R}$ & $\begin{array}{c}\text { Least } \\
\text { concern }\end{array}$ \\
\hline 30 & $\begin{array}{l}\text { Charadriifor } \\
\text { mes }\end{array}$ & Burhinidae & Great thick knee & $\begin{array}{c}\text { Esacus } \\
\text { recurvirostris }\end{array}$ & $\begin{array}{l}\text { Carnivo } \\
\text { rous }\end{array}$ & $\mathrm{W}$ & $\begin{array}{c}\text { Near } \\
\text { threatene } \\
\mathrm{d}\end{array}$ \\
\hline 31 & $\begin{array}{c}\text { Charadriifor } \\
\text { mes }\end{array}$ & Burhinidae & Indian thick knee & $\begin{array}{l}\text { Burhinus } \\
\text { oedicnemus }\end{array}$ & $\begin{array}{l}\text { Insectiv } \\
\text { orous }\end{array}$ & $\mathrm{R}$ & $\begin{array}{c}\text { Least } \\
\text { concern }\end{array}$ \\
\hline 32 & $\begin{array}{c}\text { Charadriifor } \\
\text { mes }\end{array}$ & $\begin{array}{c}\text { Charadriid } \\
\text { ae } \\
\end{array}$ & Kentish Plover & $\begin{array}{l}\text { Charadrius } \\
\text { alexandrinus }\end{array}$ & $\begin{array}{l}\text { Insectiv } \\
\text { orous }\end{array}$ & $\mathrm{W}$ & $\begin{array}{c}\text { Least } \\
\text { concern }\end{array}$ \\
\hline
\end{tabular}


Avifaunal Composition of Various Microhabitats of Southern Nagaur (Parbatsar, Kuchaman, Nawa and Makrana), Rajasthan

\begin{tabular}{|c|c|c|c|c|c|c|c|}
\hline 33 & $\begin{array}{c}\text { Charadriifor } \\
\text { mes }\end{array}$ & $\begin{array}{c}\text { Charadriid } \\
\text { ae }\end{array}$ & $\begin{array}{l}\text { Little Ringed } \\
\text { Plover }\end{array}$ & Charadrius dubius & $\begin{array}{c}\text { Insectiv } \\
\text { orous }\end{array}$ & $\mathrm{R}$ & $\begin{array}{c}\text { Least } \\
\text { concern }\end{array}$ \\
\hline 34 & $\begin{array}{c}\text { Charadriifor } \\
\text { mes }\end{array}$ & $\begin{array}{c}\text { Charadriid } \\
\text { ae }\end{array}$ & $\begin{array}{l}\text { Red wattled } \\
\text { lapwing }\end{array}$ & Vanellus indicus & $\begin{array}{c}\text { Insectiv } \\
\text { orous }\end{array}$ & $\mathrm{R}$ & $\begin{array}{c}\text { Least } \\
\text { concern }\end{array}$ \\
\hline 35 & $\begin{array}{c}\text { Charadriifor } \\
\text { mes }\end{array}$ & $\begin{array}{c}\text { Charadriid } \\
\text { ae }\end{array}$ & $\begin{array}{l}\text { Yellow wattled } \\
\text { lapwing }\end{array}$ & $\begin{array}{c}\text { Vanellus } \\
\text { malabaricus }\end{array}$ & $\begin{array}{c}\text { Insectiv } \\
\text { orous }\end{array}$ & $\mathrm{R}$ & $\begin{array}{c}\text { Least } \\
\text { concern }\end{array}$ \\
\hline 36 & $\begin{array}{c}\text { Charadriifor } \\
\text { mes }\end{array}$ & Glareolidae & Small pratincole & Glareola lactea & $\begin{array}{c}\text { Insectiv } \\
\text { orous }\end{array}$ & $\mathrm{W}$ & $\begin{array}{c}\text { Least } \\
\text { concern }\end{array}$ \\
\hline 37 & $\begin{array}{c}\text { Charadriifor } \\
\text { mes }\end{array}$ & Laridae & Black headed gull & $\begin{array}{l}\text { Chroicocephalus } \\
\text { ridibundus }\end{array}$ & $\begin{array}{l}\text { Omnivo } \\
\text { rous }\end{array}$ & $\mathrm{W}$ & $\begin{array}{c}\text { Least } \\
\text { concern }\end{array}$ \\
\hline 38 & $\begin{array}{c}\text { Charadriifor } \\
\text { mes }\end{array}$ & Laridae & Brown Headed Gull & $\begin{array}{c}\text { Chroicocephalus } \\
\text { brunnicephalus }\end{array}$ & $\begin{array}{l}\text { Omnivo } \\
\text { rous }\end{array}$ & $\mathrm{W}$ & $\begin{array}{c}\text { Least } \\
\text { concern }\end{array}$ \\
\hline 39 & $\begin{array}{c}\text { Charadriifor } \\
\text { mes }\end{array}$ & Laridae & Gull billed tern & $\begin{array}{l}\text { Gelochelidon } \\
\text { nilotica }\end{array}$ & $\begin{array}{l}\text { Carnivo } \\
\text { rous }\end{array}$ & $\mathrm{W}$ & $\begin{array}{c}\text { Least } \\
\text { concern }\end{array}$ \\
\hline 40 & $\begin{array}{c}\text { Charadriifor } \\
\text { mes }\end{array}$ & Laridae & Pallas gull & $\begin{array}{l}\text { Ichthyaetus } \\
\text { ichthyaetus }\end{array}$ & $\begin{array}{l}\text { Carnivo } \\
\text { rous }\end{array}$ & $\mathrm{W}$ & $\begin{array}{c}\text { Least } \\
\text { concern }\end{array}$ \\
\hline 41 & $\begin{array}{c}\text { Charadriifor } \\
\text { mes }\end{array}$ & Laridae & River tern & Sterna aurantia & $\begin{array}{c}\text { Carnivo } \\
\text { rous }\end{array}$ & $\mathrm{W}$ & $\begin{array}{c}\text { Near } \\
\text { Threatene } \\
\text { d }\end{array}$ \\
\hline 42 & $\begin{array}{c}\text { Charadriifor } \\
\text { mes }\end{array}$ & Laridae & Whiskered tern & Chlidonias hybrida & $\begin{array}{l}\text { Carnivo } \\
\text { rous }\end{array}$ & $\mathrm{W}$ & $\begin{array}{c}\text { Least } \\
\text { concern }\end{array}$ \\
\hline 43 & $\begin{array}{c}\text { Charadriifor } \\
\text { mes }\end{array}$ & $\begin{array}{c}\text { Recurviros } \\
\text { tridae }\end{array}$ & Black winged stilt & $\begin{array}{l}\text { Himantopus } \\
\text { himantopus }\end{array}$ & $\begin{array}{l}\text { Carnivo } \\
\text { rous }\end{array}$ & $\mathrm{R}$ & $\begin{array}{c}\text { Least } \\
\text { concern }\end{array}$ \\
\hline 44 & $\begin{array}{c}\text { Charadriifor } \\
\text { mes }\end{array}$ & $\begin{array}{l}\text { Recurviros } \\
\text { tridae }\end{array}$ & Pied avocet & $\begin{array}{c}\text { Recurvirostra } \\
\text { avosetta }\end{array}$ & $\begin{array}{c}\text { Insectiv } \\
\text { orous }\end{array}$ & $\mathrm{W}$ & $\begin{array}{c}\text { Least } \\
\text { concern }\end{array}$ \\
\hline 45 & $\begin{array}{c}\text { Charadriifor } \\
\text { mes }\end{array}$ & $\begin{array}{c}\text { Rostratulid } \\
\text { ae }\end{array}$ & $\begin{array}{l}\text { Greater painted } \\
\text { snipe }\end{array}$ & $\begin{array}{c}\text { Rostratula } \\
\text { benghalensis }\end{array}$ & $\begin{array}{l}\text { Omnivo } \\
\text { rous }\end{array}$ & $\mathrm{R}$ & $\begin{array}{c}\text { Least } \\
\text { concern }\end{array}$ \\
\hline 46 & $\begin{array}{c}\text { Charadriifor } \\
\text { mes }\end{array}$ & $\begin{array}{c}\text { Scolopacid } \\
\text { ae }\end{array}$ & Black tailed godwit & Limosa limosa & $\begin{array}{l}\text { Insectiv } \\
\text { orous }\end{array}$ & W & $\begin{array}{c}\text { Near } \\
\text { Threatene } \\
\text { d }\end{array}$ \\
\hline 47 & $\begin{array}{c}\text { Charadriifor } \\
\text { mes }\end{array}$ & $\begin{array}{c}\text { Scolopacid } \\
\text { ae }\end{array}$ & $\begin{array}{l}\text { Common } \\
\text { Sandpiper }\end{array}$ & Actitis hypoleucosa & $\begin{array}{c}\text { Insectiv } \\
\text { orous }\end{array}$ & W & $\begin{array}{c}\text { Least } \\
\text { concern }\end{array}$ \\
\hline 48 & $\begin{array}{c}\text { Charadriifor } \\
\text { mes }\end{array}$ & $\begin{array}{c}\text { Scolopacid } \\
\text { ae }\end{array}$ & Little stint & Calidris minuta & $\begin{array}{c}\text { Insectiv } \\
\text { orous }\end{array}$ & $\mathrm{W}$ & $\begin{array}{c}\text { Least } \\
\text { concern }\end{array}$ \\
\hline 49 & $\begin{array}{c}\text { Charadriifor } \\
\text { mes }\end{array}$ & $\begin{array}{c}\text { Scolopacid } \\
\text { ae }\end{array}$ & Ruff & Calidris pugnax & $\begin{array}{l}\text { Herbivo } \\
\text { rous }\end{array}$ & $\mathrm{W}$ & $\begin{array}{c}\text { Least } \\
\text { concern }\end{array}$ \\
\hline 50 & $\begin{array}{c}\text { Charadriifor } \\
\text { mes }\end{array}$ & $\begin{array}{c}\text { Scolopacid } \\
\text { ae }\end{array}$ & Common snipe & $\begin{array}{c}\text { Gallinago } \\
\text { gallinaggo }\end{array}$ & $\begin{array}{c}\text { Insectiv } \\
\text { orous }\end{array}$ & $\mathrm{W}$ & $\begin{array}{c}\text { Least } \\
\text { concern }\end{array}$ \\
\hline 51 & $\begin{array}{c}\text { Charadriifor } \\
\text { mes }\end{array}$ & $\begin{array}{c}\text { Scolopacid } \\
\text { ae }\end{array}$ & Spotted redshank & Tringa erythropus & $\begin{array}{c}\text { Insectiv } \\
\text { orous }\end{array}$ & $\mathrm{W}$ & $\begin{array}{c}\text { Least } \\
\text { concern }\end{array}$ \\
\hline 52 & $\begin{array}{c}\text { Charadriifor } \\
\text { mes }\end{array}$ & $\begin{array}{c}\text { Scolopacid } \\
\text { ae }\end{array}$ & Common redshank & Tringa totanus & $\begin{array}{c}\text { Insectiv } \\
\text { orous }\end{array}$ & $\mathrm{W}$ & $\begin{array}{c}\text { Least } \\
\text { concern }\end{array}$ \\
\hline 53 & $\begin{array}{c}\text { Charadriifor } \\
\text { mes }\end{array}$ & $\begin{array}{c}\text { Scolopacid } \\
\text { ae }\end{array}$ & Green sandpiper & Tringa ochropus & $\begin{array}{c}\text { Insectiv } \\
\text { orous }\end{array}$ & $\mathrm{W}$ & $\begin{array}{c}\text { Least } \\
\text { concern }\end{array}$ \\
\hline 54 & $\begin{array}{c}\text { Charadriifor } \\
\text { mes }\end{array}$ & $\begin{array}{c}\text { Scolopacid } \\
\text { ae }\end{array}$ & Wood sandpiper & Tringa glareola & $\begin{array}{c}\text { Insectiv } \\
\text { orous }\end{array}$ & $\mathrm{W}$ & $\begin{array}{c}\text { Least } \\
\text { concern }\end{array}$ \\
\hline 55 & $\begin{array}{c}\text { Charadriifor } \\
\text { mes }\end{array}$ & $\begin{array}{c}\text { Scolopacid } \\
\text { ae } \\
\end{array}$ & Temminck's stint & Calidris temminckii & $\begin{array}{c}\text { Insectiv } \\
\text { orous }\end{array}$ & $\mathrm{W}$ & $\begin{array}{c}\text { Least } \\
\text { concern }\end{array}$ \\
\hline 56 & $\begin{array}{c}\text { Charadriifor } \\
\text { mes }\end{array}$ & $\begin{array}{c}\text { Scolopacid } \\
\text { ae }\end{array}$ & Curlew sandpiper & Calidris ferruginea & $\begin{array}{c}\text { Insectiv } \\
\text { orous }\end{array}$ & $\mathrm{W}$ & $\begin{array}{c}\text { Least } \\
\text { concern }\end{array}$ \\
\hline
\end{tabular}


Rounak Choudhary, Praveen Mathur, and Vivek Sharma

\begin{tabular}{|c|c|c|c|c|c|c|c|}
\hline 57 & $\begin{array}{c}\text { Charadriifor } \\
\text { mes }\end{array}$ & Turnicidae & $\begin{array}{l}\text { Barred button } \\
\text { quail }\end{array}$ & Turnix suscitator & $\begin{array}{l}\text { Granivo } \\
\text { rous }\end{array}$ & $\mathrm{R}$ & $\begin{array}{c}\text { Least } \\
\text { concern }\end{array}$ \\
\hline 58 & $\begin{array}{l}\text { Ciconiiforme } \\
\mathrm{s}\end{array}$ & Ciconiidae & Asian openbill & Anastomus oscitans & $\begin{array}{l}\text { Carnivo } \\
\text { rous }\end{array}$ & $\mathrm{R}$ & $\begin{array}{c}\text { Least } \\
\text { concern }\end{array}$ \\
\hline 59 & $\begin{array}{c}\text { Ciconiiforme } \\
\mathrm{s}\end{array}$ & Ciconiidae & Painted stork & $\begin{array}{c}\text { Mycteria } \\
\text { leucocephala }\end{array}$ & $\begin{array}{l}\text { Carnivo } \\
\text { rous }\end{array}$ & $\mathrm{R}$ & $\begin{array}{c}\text { Near } \\
\text { Threatene } \\
d\end{array}$ \\
\hline 60 & $\begin{array}{c}\text { Ciconiiforme } \\
\mathrm{s} \\
\end{array}$ & Ciconiidae & $\begin{array}{c}\text { Woolly necked } \\
\text { stork }\end{array}$ & Ciconia episcopus & $\begin{array}{l}\text { Carnivo } \\
\text { rous }\end{array}$ & $\mathrm{R}$ & $\begin{array}{c}\text { Vulnerabl } \\
\text { e }\end{array}$ \\
\hline 61 & $\begin{array}{l}\text { Columbifor } \\
\text { mes }\end{array}$ & $\begin{array}{c}\text { Columbida } \\
\text { e }\end{array}$ & Blue rock dove & Columba livia & $\begin{array}{l}\text { Granivo } \\
\text { rous }\end{array}$ & $\mathrm{R}$ & $\begin{array}{c}\text { Least } \\
\text { concern }\end{array}$ \\
\hline 62 & $\begin{array}{c}\text { Columbifor } \\
\text { mes }\end{array}$ & $\begin{array}{c}\text { Columbida } \\
\text { e }\end{array}$ & Laughing Dove & $\begin{array}{c}\text { Spilopelia } \\
\text { senegalensis }\end{array}$ & $\begin{array}{l}\text { Granivo } \\
\text { rous }\end{array}$ & $\mathrm{R}$ & $\begin{array}{c}\text { Least } \\
\text { concern }\end{array}$ \\
\hline 63 & $\begin{array}{c}\text { Columbifor } \\
\text { mes }\end{array}$ & $\begin{array}{c}\text { Columbida } \\
\text { e }\end{array}$ & Spotted dove & Spilopelia chinensis & $\begin{array}{l}\text { Granivo } \\
\text { rous }\end{array}$ & $\mathrm{R}$ & $\begin{array}{c}\text { Least } \\
\text { concern }\end{array}$ \\
\hline 64 & $\begin{array}{l}\text { Columbifor } \\
\text { mes }\end{array}$ & $\begin{array}{c}\text { Columbida } \\
\text { e }\end{array}$ & Red-collared dove & $\begin{array}{c}\text { Streptopelia } \\
\text { tranquebarica }\end{array}$ & $\begin{array}{l}\text { Granivo } \\
\text { rous }\end{array}$ & $\mathrm{R}$ & $\begin{array}{c}\text { Least } \\
\text { concern }\end{array}$ \\
\hline 65 & $\begin{array}{c}\text { Columbifor } \\
\text { mes }\end{array}$ & $\begin{array}{c}\text { Columbida } \\
\text { e }\end{array}$ & $\begin{array}{c}\text { Eurasian collared } \\
\text { dove }\end{array}$ & $\begin{array}{l}\text { Streptopelia } \\
\text { decaocto }\end{array}$ & $\begin{array}{l}\text { Granivo } \\
\text { rous }\end{array}$ & $\mathrm{R}$ & $\begin{array}{c}\text { Least } \\
\text { concern }\end{array}$ \\
\hline 66 & $\begin{array}{c}\text { Columbifor } \\
\text { mes }\end{array}$ & $\begin{array}{c}\text { Columbida } \\
\text { e }\end{array}$ & $\begin{array}{l}\text { Yellow footed } \\
\text { green pigeoon }\end{array}$ & $\begin{array}{c}\text { Treron } \\
\text { phoenicoptera }\end{array}$ & $\begin{array}{l}\text { Frugivo } \\
\text { rous }\end{array}$ & $\mathrm{R}$ & $\begin{array}{c}\text { Least } \\
\text { concern }\end{array}$ \\
\hline 67 & $\begin{array}{c}\text { Coraciiforme } \\
\mathrm{s}\end{array}$ & $\begin{array}{c}\text { Alcedinida } \\
\mathrm{e}\end{array}$ & Common kingfisher & Alcedo atthis & $\begin{array}{l}\text { Carnivo } \\
\text { rous }\end{array}$ & $\mathrm{R}$ & $\begin{array}{c}\text { Least } \\
\text { concern }\end{array}$ \\
\hline 68 & $\begin{array}{c}\text { Coraciiforme } \\
\text { s } \\
\end{array}$ & $\begin{array}{c}\text { Alcedinida } \\
\text { e }\end{array}$ & Pied kingfisher & Ceryle rudis & $\begin{array}{l}\text { Carnivo } \\
\text { rous }\end{array}$ & $\mathrm{R}$ & $\begin{array}{c}\text { Least } \\
\text { concern }\end{array}$ \\
\hline 69 & $\begin{array}{c}\text { Coraciiforme } \\
\text { s }\end{array}$ & $\begin{array}{l}\text { Alcedinida } \\
\text { e }\end{array}$ & $\begin{array}{l}\text { White throated } \\
\text { kingfisher }\end{array}$ & Halcyon smyrnensis & $\begin{array}{l}\text { Insectiv } \\
\text { orous }\end{array}$ & $\mathrm{R}$ & $\begin{array}{c}\text { Least } \\
\text { concern }\end{array}$ \\
\hline 70 & $\begin{array}{c}\text { Coraciiforme } \\
\text { s }\end{array}$ & Coraciidae & European roller & Coracias garrulus & $\begin{array}{l}\text { Insectiv } \\
\text { orous }\end{array}$ & $\mathrm{S}$ & $\begin{array}{c}\text { Least } \\
\text { concern }\end{array}$ \\
\hline 71 & $\begin{array}{c}\text { Coraciiforme } \\
\text { s }\end{array}$ & Coraciidae & Indian roller & $\begin{array}{c}\text { Coracias } \\
\text { benghalensis }\end{array}$ & $\begin{array}{l}\text { Insectiv } \\
\text { orous }\end{array}$ & $\mathrm{R}$ & $\begin{array}{c}\text { Least } \\
\text { concern }\end{array}$ \\
\hline 72 & $\begin{array}{c}\text { Coraciiforme } \\
\mathrm{s} \\
\end{array}$ & Meropidae & Green bee-eater & Merops orientalis & $\begin{array}{l}\text { Insectiv } \\
\text { orous }\end{array}$ & $\mathrm{R}$ & $\begin{array}{c}\text { Least } \\
\text { concern }\end{array}$ \\
\hline 73 & $\begin{array}{c}\text { Coraciiforme } \\
\text { s } \\
\end{array}$ & Meropidae & $\begin{array}{l}\text { Blue tailed bee- } \\
\text { eater }\end{array}$ & Merops philippinus & $\begin{array}{l}\text { Insectiv } \\
\text { orous }\end{array}$ & $\mathrm{W}$ & $\begin{array}{c}\text { Least } \\
\text { concern }\end{array}$ \\
\hline 74 & $\begin{array}{c}\text { Coraciiforme } \\
\text { s }\end{array}$ & Meropidae & $\begin{array}{l}\text { Blue cheeked bee- } \\
\text { eater }\end{array}$ & Merops persicus & $\begin{array}{l}\text { Insectiv } \\
\text { orous }\end{array}$ & $\mathrm{W}$ & $\begin{array}{l}\text { Least } \\
\text { concern }\end{array}$ \\
\hline 75 & $\begin{array}{c}\text { Cuculiforme } \\
\text { s }\end{array}$ & Cuculidae & Jacobin cuckoo & Clamator jacobinus & $\begin{array}{l}\text { Insectiv } \\
\text { orous }\end{array}$ & $\mathrm{S}$ & $\begin{array}{c}\text { Least } \\
\text { concern }\end{array}$ \\
\hline 76 & $\begin{array}{c}\text { Cuculiforme } \\
\mathrm{s} \\
\end{array}$ & Cuculidae & $\begin{array}{l}\text { Common hawk } \\
\text { cuckoo }\end{array}$ & Hierococcyx varius & $\begin{array}{l}\text { Insectiv } \\
\text { orous }\end{array}$ & $\mathrm{R}$ & $\begin{array}{c}\text { Least } \\
\text { concern }\end{array}$ \\
\hline 77 & $\begin{array}{c}\text { Cuculiforme } \\
\text { s }\end{array}$ & Cuculidae & Asian koel & $\begin{array}{l}\text { Eudynamys } \\
\text { scolopaceus }\end{array}$ & $\begin{array}{l}\text { Frugivo } \\
\text { rous }\end{array}$ & $\mathrm{R}$ & $\begin{array}{c}\text { Least } \\
\text { concern }\end{array}$ \\
\hline 78 & $\begin{array}{c}\text { Cuculiforme } \\
\mathrm{s}\end{array}$ & Cuculidae & Greater coucal & Centropus sinensis & $\begin{array}{l}\text { Carnivo } \\
\text { rous }\end{array}$ & $\mathrm{R}$ & $\begin{array}{c}\text { Least } \\
\text { concern }\end{array}$ \\
\hline 79 & $\begin{array}{c}\text { Falconiform } \\
\text { es }\end{array}$ & Falconidae & Common kestrel & Falco tinnunculus & $\begin{array}{l}\text { Carnivo } \\
\text { rous }\end{array}$ & $\mathrm{R}$ & $\begin{array}{c}\text { Least } \\
\text { concern }\end{array}$ \\
\hline 80 & Galliformes & $\begin{array}{c}\text { Phasianida } \\
\text { e }\end{array}$ & Grey Francolin & $\begin{array}{c}\text { Francolinus } \\
\text { pondicerianus }\end{array}$ & $\begin{array}{l}\text { Granivo } \\
\text { rous }\end{array}$ & $\mathrm{R}$ & $\begin{array}{c}\text { Least } \\
\text { concern }\end{array}$ \\
\hline 81 & Galliformes & $\begin{array}{c}\text { Phasianida } \\
\text { e }\end{array}$ & Common quail & Coturnix coturnix & $\begin{array}{l}\text { Granivo } \\
\text { rous }\end{array}$ & $S$ & $\begin{array}{c}\text { Least } \\
\text { concern }\end{array}$ \\
\hline
\end{tabular}


Avifaunal Composition of Various Microhabitats of Southern Nagaur (Parbatsar, Kuchaman, Nawa and Makrana), Rajasthan

\begin{tabular}{|c|c|c|c|c|c|c|c|}
\hline 82 & Galliformes & $\begin{array}{c}\text { Phasianida } \\
\text { e }\end{array}$ & Rain quail & $\begin{array}{c}\text { Coturnix } \\
\text { coromandelica }\end{array}$ & $\begin{array}{l}\text { Granivo } \\
\text { rous }\end{array}$ & $\mathrm{R}$ & $\begin{array}{c}\text { Least } \\
\text { concern }\end{array}$ \\
\hline 83 & Galliformes & $\begin{array}{c}\text { Phasianida } \\
\text { e }\end{array}$ & Indian peafowl & Pavo cristatus & $\begin{array}{c}\text { Omnivo } \\
\text { rous }\end{array}$ & $\mathrm{R}$ & $\begin{array}{c}\text { Least } \\
\text { concern }\end{array}$ \\
\hline 84 & Galliformes & $\begin{array}{c}\text { Phasianida } \\
\text { e }\end{array}$ & Rock bush quail & $\begin{array}{l}\text { Perdicula } \\
\text { argoondha }\end{array}$ & $\begin{array}{l}\text { Herbivo } \\
\text { rous }\end{array}$ & $\mathrm{R}$ & $\begin{array}{c}\text { Least } \\
\text { concern }\end{array}$ \\
\hline 85 & Gruiformes & Gruidae & Common crane & Grus grus & $\begin{array}{l}\text { Omnivo } \\
\text { rous }\end{array}$ & $\mathrm{W}$ & $\begin{array}{c}\text { Least } \\
\text { concern }\end{array}$ \\
\hline 86 & Gruiformes & Gruidae & Demoiselle crane & Grus virgo & $\begin{array}{l}\text { Omnivo } \\
\text { rous }\end{array}$ & $\mathrm{W}$ & $\begin{array}{c}\text { Least } \\
\text { concern }\end{array}$ \\
\hline 87 & Gruiformes & Rallidae & $\begin{array}{c}\text { White breasted } \\
\text { water hen }\end{array}$ & $\begin{array}{l}\text { Amaurornis } \\
\text { phoenicurus }\end{array}$ & $\begin{array}{l}\text { Insectiv } \\
\text { orous }\end{array}$ & $\mathrm{W}$ & $\begin{array}{c}\text { Least } \\
\text { concern }\end{array}$ \\
\hline 88 & Gruiformes & Rallidae & $\begin{array}{l}\text { Grey headed } \\
\text { swamp hen }\end{array}$ & $\begin{array}{c}\text { Porphyrio } \\
\text { poliocephalus }\end{array}$ & $\begin{array}{l}\text { Omnivo } \\
\text { rous }\end{array}$ & $\mathrm{R}$ & $\begin{array}{c}\text { Least } \\
\text { concern }\end{array}$ \\
\hline 89 & Gruiformes & Rallidae & Common moorhen & Gallinula chloropus & $\begin{array}{l}\text { Omnivo } \\
\text { rous }\end{array}$ & $\mathrm{R}$ & $\begin{array}{c}\text { Least } \\
\text { concern }\end{array}$ \\
\hline 90 & Gruiformes & Rallidae & Common coot & Fulica atra & $\begin{array}{l}\text { Omnivo } \\
\text { rous }\end{array}$ & $\mathrm{R}$ & $\begin{array}{c}\text { Least } \\
\text { concern }\end{array}$ \\
\hline 91 & $\begin{array}{l}\text { Passeriform } \\
\text { es }\end{array}$ & Alaudidae & Indian bushlark & $\begin{array}{c}\text { Mirafra } \\
\text { erythroptera }\end{array}$ & $\begin{array}{l}\text { Granivo } \\
\text { rous }\end{array}$ & $\mathrm{R}$ & $\begin{array}{c}\text { Least } \\
\text { concern }\end{array}$ \\
\hline 92 & $\begin{array}{l}\text { Passeriform } \\
\text { es }\end{array}$ & Alaudidae & Crested lark & Galerida cristata & $\begin{array}{l}\text { Granivo } \\
\text { rous }\end{array}$ & $\mathrm{R}$ & $\begin{array}{c}\text { Least } \\
\text { concern }\end{array}$ \\
\hline 93 & $\begin{array}{c}\text { Passeriform } \\
\text { es }\end{array}$ & Alaudidae & Rufous tailed lark & $\begin{array}{l}\text { Ammomanes } \\
\text { phoenicura }\end{array}$ & $\begin{array}{l}\text { Granivo } \\
\text { rous }\end{array}$ & $\mathrm{R}$ & $\begin{array}{c}\text { Least } \\
\text { concern }\end{array}$ \\
\hline 94 & $\begin{array}{c}\text { Passeriform } \\
\text { es }\end{array}$ & Alaudidae & Singing bushlark & Mirafra cantillans & $\begin{array}{l}\text { Granivo } \\
\text { rous }\end{array}$ & $\mathrm{R}$ & $\begin{array}{c}\text { Least } \\
\text { concern }\end{array}$ \\
\hline 95 & $\begin{array}{c}\text { Passeriform } \\
\text { es }\end{array}$ & Alaudidae & $\begin{array}{l}\text { Greater short toed } \\
\text { lark }\end{array}$ & $\begin{array}{c}\text { Calandrella } \\
\text { brachydactyla }\end{array}$ & $\begin{array}{l}\text { Granivo } \\
\text { rous }\end{array}$ & $\mathrm{W}$ & $\begin{array}{c}\text { Least } \\
\text { concern }\end{array}$ \\
\hline 96 & $\begin{array}{c}\text { Passeriform } \\
\text { es }\end{array}$ & Alaudidae & $\begin{array}{l}\text { Ashy crowned } \\
\text { sparrow lark }\end{array}$ & Eremopterix griseus & $\begin{array}{l}\text { Granivo } \\
\text { rous }\end{array}$ & $\mathrm{R}$ & $\begin{array}{c}\text { Least } \\
\text { concern }\end{array}$ \\
\hline 97 & $\begin{array}{c}\text { Passeriform } \\
\text { es }\end{array}$ & $\begin{array}{l}\text { Campepha } \\
\text { gidae }\end{array}$ & Small minivete & $\begin{array}{l}\text { Pericrocotus } \\
\text { cinnamomeus }\end{array}$ & $\begin{array}{l}\text { Insectiv } \\
\text { orous }\end{array}$ & $\mathrm{R}$ & $\begin{array}{c}\text { Least } \\
\text { concern }\end{array}$ \\
\hline 98 & $\begin{array}{c}\text { Passeriform } \\
\text { es }\end{array}$ & $\begin{array}{l}\text { Campepha } \\
\text { gidae }\end{array}$ & $\begin{array}{l}\text { Large cuckoo } \\
\text { shrike }\end{array}$ & Coracina macei & $\begin{array}{l}\text { Insectiv } \\
\text { orous }\end{array}$ & $\mathrm{W}$ & $\begin{array}{c}\text { Least } \\
\text { concern }\end{array}$ \\
\hline 99 & $\begin{array}{c}\text { Passeriform } \\
\text { es }\end{array}$ & Certhiidae & $\begin{array}{c}\text { Indian spotted } \\
\text { creeper }\end{array}$ & Salpornis spilonotus & $\begin{array}{l}\text { Insectiv } \\
\text { orous }\end{array}$ & $\mathrm{R}$ & $\begin{array}{c}\text { Least } \\
\text { concern }\end{array}$ \\
\hline $\begin{array}{c}10 \\
0 \\
\end{array}$ & $\begin{array}{c}\text { Passeriform } \\
\text { es }\end{array}$ & $\begin{array}{c}\text { Cisticolida } \\
\mathrm{e}\end{array}$ & $\begin{array}{c}\text { Rufous fronted } \\
\text { prinia }\end{array}$ & Prinia buchanani & $\begin{array}{l}\text { Insectiv } \\
\text { orous }\end{array}$ & $\mathrm{R}$ & $\begin{array}{c}\text { Least } \\
\text { concern }\end{array}$ \\
\hline $\begin{array}{c}10 \\
1\end{array}$ & $\begin{array}{c}\text { Passeriform } \\
\text { es }\end{array}$ & $\begin{array}{c}\text { Cisticolida } \\
\text { e }\end{array}$ & Plain prinia & Prinia inornata & $\begin{array}{l}\text { Insectiv } \\
\text { orous }\end{array}$ & $\mathrm{R}$ & $\begin{array}{c}\text { Least } \\
\text { concern }\end{array}$ \\
\hline $\begin{array}{c}10 \\
2\end{array}$ & $\begin{array}{c}\text { Passeriform } \\
\text { es }\end{array}$ & $\begin{array}{c}\text { Cisticolida } \\
\text { e }\end{array}$ & Ashy prinia & Prinia socialis & $\begin{array}{l}\text { Insectiv } \\
\text { orous }\end{array}$ & $\mathrm{R}$ & $\begin{array}{c}\text { Least } \\
\text { concern }\end{array}$ \\
\hline $\begin{array}{c}10 \\
3\end{array}$ & $\begin{array}{c}\text { Passeriform } \\
\text { es }\end{array}$ & $\begin{array}{c}\text { Cisticolida } \\
\text { e }\end{array}$ & $\begin{array}{l}\text { Grey breasted } \\
\text { prinia }\end{array}$ & Prinia hodgsonii & $\begin{array}{l}\text { Insectiv } \\
\text { orous }\end{array}$ & $\mathrm{R}$ & $\begin{array}{c}\text { Least } \\
\text { concern }\end{array}$ \\
\hline $\begin{array}{c}10 \\
4\end{array}$ & $\begin{array}{c}\text { Passeriform } \\
\text { es }\end{array}$ & $\begin{array}{c}\text { Cisticolida } \\
\text { e }\end{array}$ & Jungle prina & Prinia sylvatica & $\begin{array}{l}\text { Insectiv } \\
\text { orous }\end{array}$ & $\mathrm{R}$ & $\begin{array}{c}\text { Least } \\
\text { concern }\end{array}$ \\
\hline $\begin{array}{c}10 \\
5\end{array}$ & $\begin{array}{c}\text { Passeriform } \\
\text { es }\end{array}$ & $\begin{array}{c}\text { Cisticolida } \\
\text { e }\end{array}$ & Common tailorbird & $\begin{array}{l}\text { Orthotomus } \\
\text { sutorius }\end{array}$ & $\begin{array}{l}\text { Insectiv } \\
\text { orous }\end{array}$ & $\mathrm{R}$ & $\begin{array}{c}\text { Least } \\
\text { concern }\end{array}$ \\
\hline $\begin{array}{c}10 \\
6 \\
\end{array}$ & $\begin{array}{c}\text { Passeriform } \\
\text { es } \\
\end{array}$ & Corvidae & Rufous treepie & $\begin{array}{c}\text { Dendrocitta } \\
\text { vagabunda }\end{array}$ & $\begin{array}{c}\text { Omnivo } \\
\text { rous }\end{array}$ & $\mathrm{R}$ & $\begin{array}{c}\text { Least } \\
\text { concern }\end{array}$ \\
\hline
\end{tabular}


Rounak Choudhary, Praveen Mathur, and Vivek Sharma

\begin{tabular}{|c|c|c|c|c|c|c|c|}
\hline $\begin{array}{c}10 \\
7\end{array}$ & $\begin{array}{c}\text { Passeriform } \\
\text { es }\end{array}$ & Corvidae & House crow & Corvus splendens & $\begin{array}{l}\text { Omnivo } \\
\text { rous }\end{array}$ & $\mathrm{R}$ & $\begin{array}{c}\text { Least } \\
\text { concern }\end{array}$ \\
\hline $\begin{array}{c}10 \\
8\end{array}$ & $\begin{array}{c}\text { Passeriform } \\
\text { es }\end{array}$ & Dicruridae & Black drongo & $\begin{array}{c}\text { Dicrurus } \\
\text { macrocercus }\end{array}$ & $\begin{array}{c}\text { Insectiv } \\
\text { orous }\end{array}$ & $\mathrm{R}$ & $\begin{array}{c}\text { Least } \\
\text { concern }\end{array}$ \\
\hline $\begin{array}{c}10 \\
9\end{array}$ & $\begin{array}{c}\text { Passeriform } \\
\text { es }\end{array}$ & Dicruridae & $\begin{array}{c}\text { White bellied } \\
\text { drongo }\end{array}$ & $\begin{array}{c}\text { Dicrurus } \\
\text { caerulescens }\end{array}$ & $\begin{array}{c}\text { Insectiv } \\
\text { orous }\end{array}$ & $\mathrm{R}$ & $\begin{array}{c}\text { Least } \\
\text { concern }\end{array}$ \\
\hline $\begin{array}{c}11 \\
0\end{array}$ & $\begin{array}{c}\text { Passeriform } \\
\text { es }\end{array}$ & $\begin{array}{c}\text { Emberizid } \\
\text { ae }\end{array}$ & Crested bunting & Emberiza lathami & $\begin{array}{l}\text { Granivo } \\
\text { rous }\end{array}$ & $\mathrm{R}$ & $\begin{array}{c}\text { Least } \\
\text { concern }\end{array}$ \\
\hline $\begin{array}{c}11 \\
1\end{array}$ & $\begin{array}{c}\text { Passeriform } \\
\text { es }\end{array}$ & $\begin{array}{c}\text { Emberizid } \\
\text { ae }\end{array}$ & $\begin{array}{l}\text { Red headed } \\
\text { bunting }\end{array}$ & Emberiza bruniceps & $\begin{array}{c}\text { Granivo } \\
\text { rous }\end{array}$ & $\mathrm{W}$ & $\begin{array}{c}\text { Least } \\
\text { concern }\end{array}$ \\
\hline $\begin{array}{c}11 \\
2\end{array}$ & $\begin{array}{c}\text { Passeriform } \\
\text { es }\end{array}$ & Estrildidae & Indian silverbill & Euodice malabarica & $\begin{array}{c}\text { Granivo } \\
\text { rous }\end{array}$ & $\mathrm{R}$ & $\begin{array}{c}\text { Least } \\
\text { concern }\end{array}$ \\
\hline $\begin{array}{c}11 \\
3\end{array}$ & $\begin{array}{c}\text { Passeriform } \\
\text { es }\end{array}$ & $\begin{array}{c}\text { Hirundinid } \\
\text { ae }\end{array}$ & Dusky crag martin & Hirundo concolor & $\begin{array}{c}\text { Insectiv } \\
\text { orous }\end{array}$ & $\mathrm{R}$ & $\begin{array}{c}\text { Least } \\
\text { concern }\end{array}$ \\
\hline $\begin{array}{c}11 \\
4\end{array}$ & $\begin{array}{c}\text { Passeriform } \\
\text { es }\end{array}$ & $\begin{array}{c}\text { Hirundinid } \\
\text { ae }\end{array}$ & $\begin{array}{c}\text { Streak throated } \\
\text { swallow }\end{array}$ & Hirundo fluvicola & $\begin{array}{c}\text { Insectiv } \\
\text { orous }\end{array}$ & $\mathrm{R}$ & $\begin{array}{c}\text { Least } \\
\text { concern }\end{array}$ \\
\hline $\begin{array}{c}11 \\
5\end{array}$ & $\begin{array}{c}\text { Passeriform } \\
\text { es }\end{array}$ & $\begin{array}{c}\text { Hirundinid } \\
\text { ae }\end{array}$ & $\begin{array}{c}\text { Wire tailed } \\
\text { swallow }\end{array}$ & Hirundo smithii & $\begin{array}{c}\text { Insectiv } \\
\text { orous }\end{array}$ & $\mathrm{R}$ & $\begin{array}{c}\text { Least } \\
\text { concern }\end{array}$ \\
\hline $\begin{array}{c}11 \\
6\end{array}$ & $\begin{array}{c}\text { Passeriform } \\
\text { es }\end{array}$ & $\begin{array}{c}\text { Hirundinid } \\
\text { ae }\end{array}$ & $\begin{array}{l}\text { Red Rumped } \\
\text { Swallow }\end{array}$ & Hirundo daurica & $\begin{array}{c}\text { Insectiv } \\
\text { orous }\end{array}$ & $\mathrm{R}$ & $\begin{array}{c}\text { Least } \\
\text { concern }\end{array}$ \\
\hline $\begin{array}{c}11 \\
7\end{array}$ & $\begin{array}{c}\text { Passeriform } \\
\text { es }\end{array}$ & Laniidae & Bay backed shrike & Lanius vittatus & $\begin{array}{c}\text { Insectiv } \\
\text { orous }\end{array}$ & $\mathrm{R}$ & $\begin{array}{c}\text { Least } \\
\text { concern }\end{array}$ \\
\hline $\begin{array}{c}11 \\
8\end{array}$ & $\begin{array}{c}\text { Passeriform } \\
\text { es }\end{array}$ & Laniidae & Long tailed shrike & Lanius schach & $\begin{array}{c}\text { Insectiv } \\
\text { orous }\end{array}$ & $\mathrm{R}$ & $\begin{array}{c}\text { Least } \\
\text { concern }\end{array}$ \\
\hline $\begin{array}{c}11 \\
9\end{array}$ & $\begin{array}{c}\text { Passeriform } \\
\text { es }\end{array}$ & Laniidae & $\begin{array}{c}\text { Southern grey } \\
\text { shrike }\end{array}$ & Lanius excubitor & $\begin{array}{c}\text { Insectiv } \\
\text { orous }\end{array}$ & $\mathrm{R}$ & $\begin{array}{c}\text { Vulnerabl } \\
\mathrm{e}\end{array}$ \\
\hline $\begin{array}{c}12 \\
0\end{array}$ & $\begin{array}{c}\text { Passeriform } \\
\text { es }\end{array}$ & $\begin{array}{c}\text { Leiothrichi } \\
\text { dae }\end{array}$ & Common babbler & Argya caudata & $\begin{array}{c}\text { Granivo } \\
\text { rous }\end{array}$ & $\mathrm{R}$ & $\begin{array}{c}\text { Least } \\
\text { concern }\end{array}$ \\
\hline $\begin{array}{c}12 \\
1\end{array}$ & $\begin{array}{c}\text { Passeriform } \\
\text { es }\end{array}$ & $\begin{array}{c}\text { Leiothrichi } \\
\text { dae }\end{array}$ & Large grey babbler & Turdoides malcolmi & $\begin{array}{c}\text { Granivo } \\
\text { rous }\end{array}$ & $\mathrm{R}$ & $\begin{array}{c}\text { Least } \\
\text { concern }\end{array}$ \\
\hline $\begin{array}{c}12 \\
2\end{array}$ & $\begin{array}{c}\text { Passeriform } \\
\text { es }\end{array}$ & $\begin{array}{c}\text { Leiothrichi } \\
\text { dae }\end{array}$ & Jungle babbler & Argya striata & $\begin{array}{c}\text { Granivo } \\
\text { rous }\end{array}$ & $\mathrm{R}$ & $\begin{array}{c}\text { Least } \\
\text { concern }\end{array}$ \\
\hline $\begin{array}{c}12 \\
3\end{array}$ & $\begin{array}{c}\text { Passeriform } \\
\text { es }\end{array}$ & $\begin{array}{c}\text { Motacillida } \\
\text { e }\end{array}$ & White wagtail & Motacilla alba & $\begin{array}{c}\text { Insectiv } \\
\text { orous }\end{array}$ & $\mathrm{W}$ & $\begin{array}{c}\text { Least } \\
\text { concern }\end{array}$ \\
\hline $\begin{array}{c}12 \\
4\end{array}$ & $\begin{array}{c}\text { Passeriform } \\
\text { es }\end{array}$ & $\begin{array}{c}\text { Motacillida } \\
\text { e }\end{array}$ & $\begin{array}{c}\text { White browed } \\
\text { wagtail }\end{array}$ & $\begin{array}{c}\text { Motacilla } \\
\text { maderaspatensis }\end{array}$ & $\begin{array}{c}\text { Insectiv } \\
\text { orous }\end{array}$ & $\mathrm{R}$ & $\begin{array}{c}\text { Least } \\
\text { concern }\end{array}$ \\
\hline $\begin{array}{c}12 \\
5\end{array}$ & $\begin{array}{c}\text { Passeriform } \\
\text { es }\end{array}$ & $\begin{array}{c}\text { Motacillida } \\
\mathrm{e}\end{array}$ & Citrine wagtail & Motacilla citreola & $\begin{array}{c}\text { Insectiv } \\
\text { orous }\end{array}$ & $\mathrm{W}$ & $\begin{array}{c}\text { Least } \\
\text { concern }\end{array}$ \\
\hline $\begin{array}{c}12 \\
6 \\
\end{array}$ & $\begin{array}{c}\text { Passeriform } \\
\text { es } \\
\end{array}$ & $\begin{array}{c}\text { Motacillida } \\
\text { e }\end{array}$ & Yellow wagtail & Motacilla flava & $\begin{array}{c}\text { Insectiv } \\
\text { orous }\end{array}$ & $\mathrm{W}$ & $\begin{array}{c}\text { Least } \\
\text { concern }\end{array}$ \\
\hline $\begin{array}{c}12 \\
7\end{array}$ & $\begin{array}{c}\text { Passeriform } \\
\text { es }\end{array}$ & $\begin{array}{c}\text { Motacillida } \\
\text { e }\end{array}$ & Paddyfield pipet & Anthus rufulus & $\begin{array}{c}\text { Insectiv } \\
\text { orous }\end{array}$ & $\mathrm{R}$ & $\begin{array}{c}\text { Least } \\
\text { concern }\end{array}$ \\
\hline $\begin{array}{c}12 \\
8\end{array}$ & $\begin{array}{c}\text { Passeriform } \\
\text { es }\end{array}$ & $\begin{array}{c}\text { Muscicapid } \\
\text { ae }\end{array}$ & $\begin{array}{l}\text { Red-breasted } \\
\text { flycatcher }\end{array}$ & Ficedula parva & $\begin{array}{c}\text { Insectiv } \\
\text { orous }\end{array}$ & $\mathrm{W}$ & $\begin{array}{c}\text { Least } \\
\text { concern }\end{array}$ \\
\hline $\begin{array}{c}12 \\
9\end{array}$ & $\begin{array}{c}\text { Passeriform } \\
\text { es }\end{array}$ & $\begin{array}{c}\text { Muscicapid } \\
\text { ae }\end{array}$ & Bluethroat & Luscinia svecica & $\begin{array}{c}\text { Insectiv } \\
\text { orous }\end{array}$ & $\mathrm{W}$ & $\begin{array}{c}\text { Least } \\
\text { concern }\end{array}$ \\
\hline $\begin{array}{c}13 \\
0\end{array}$ & $\begin{array}{c}\text { Passeriform } \\
\text { es }\end{array}$ & $\begin{array}{c}\text { Muscicapid } \\
\text { ae }\end{array}$ & $\begin{array}{l}\text { Oriental magpie } \\
\text { robin }\end{array}$ & Copsychus saularis & $\begin{array}{l}\text { Insectiv } \\
\text { orous }\end{array}$ & $\mathrm{R}$ & $\begin{array}{c}\text { Least } \\
\text { concern }\end{array}$ \\
\hline $\begin{array}{c}13 \\
1\end{array}$ & $\begin{array}{c}\text { Passeriform } \\
\text { es }\end{array}$ & $\begin{array}{c}\text { Muscicapid } \\
\text { ae }\end{array}$ & Indian robin & Copsychus fulicatus & $\begin{array}{c}\text { Insectiv } \\
\text { orous }\end{array}$ & $\mathrm{R}$ & $\begin{array}{c}\text { Least } \\
\text { concern }\end{array}$ \\
\hline
\end{tabular}


Avifaunal Composition of Various Microhabitats of Southern Nagaur (Parbatsar, Kuchaman, Nawa and Makrana),

Rajasthan

\begin{tabular}{|c|c|c|c|c|c|c|c|}
\hline $\begin{array}{c}13 \\
2\end{array}$ & $\begin{array}{c}\text { Passeriform } \\
\text { es }\end{array}$ & $\begin{array}{c}\text { Muscicapid } \\
\text { ae }\end{array}$ & Black redstart & $\begin{array}{c}\text { Phoenicurus } \\
\text { ochruros }\end{array}$ & $\begin{array}{c}\text { Insectiv } \\
\text { orous }\end{array}$ & $\mathrm{W}$ & $\begin{array}{c}\text { Least } \\
\text { concern }\end{array}$ \\
\hline $\begin{array}{c}13 \\
3\end{array}$ & $\begin{array}{c}\text { Passeriform } \\
\text { es }\end{array}$ & $\begin{array}{c}\text { Muscicapid } \\
\text { ae }\end{array}$ & Common Stonechat & Saxicola maurus & $\begin{array}{c}\text { Insectiv } \\
\text { orous }\end{array}$ & $\mathrm{R}$ & $\begin{array}{c}\text { Least } \\
\text { concern }\end{array}$ \\
\hline $\begin{array}{c}13 \\
4\end{array}$ & $\begin{array}{c}\text { Passeriform } \\
\text { es }\end{array}$ & $\begin{array}{c}\text { Muscicapid } \\
\text { ae }\end{array}$ & Desert wheatear & Oenanthe deserti & $\begin{array}{c}\text { Insectiv } \\
\text { orous }\end{array}$ & W & $\begin{array}{c}\text { Least } \\
\text { concern }\end{array}$ \\
\hline $\begin{array}{c}13 \\
5\end{array}$ & $\begin{array}{c}\text { Passeriform } \\
\text { es }\end{array}$ & $\begin{array}{c}\text { Muscicapid } \\
\text { ae }\end{array}$ & $\begin{array}{l}\text { Isabelline } \\
\text { wheatear }\end{array}$ & Oenanthe isabellina & $\begin{array}{c}\text { Insectiv } \\
\text { orous }\end{array}$ & W & $\begin{array}{c}\text { Least } \\
\text { concern }\end{array}$ \\
\hline $\begin{array}{c}13 \\
6\end{array}$ & $\begin{array}{c}\text { Passeriform } \\
\text { es }\end{array}$ & $\begin{array}{c}\text { Muscicapid } \\
\text { ae }\end{array}$ & Variable wheatear & Oenanthe picata & $\begin{array}{c}\text { Insectiv } \\
\text { orous }\end{array}$ & W & $\begin{array}{c}\text { Least } \\
\text { concern }\end{array}$ \\
\hline $\begin{array}{c}13 \\
7 \\
\end{array}$ & $\begin{array}{c}\text { Passeriform } \\
\text { es }\end{array}$ & $\begin{array}{c}\text { Muscicapid } \\
\text { ae }\end{array}$ & Pied bushchat & Saxicola caprata & $\begin{array}{c}\text { Insectiv } \\
\text { orous }\end{array}$ & W & $\begin{array}{c}\text { Least } \\
\text { concern }\end{array}$ \\
\hline $\begin{array}{c}13 \\
8\end{array}$ & $\begin{array}{c}\text { Passeriform } \\
\text { es }\end{array}$ & $\begin{array}{c}\text { Muscicapid } \\
\text { ae }\end{array}$ & Brown rockchat & Oenanthe fusca & $\begin{array}{c}\text { Insectiv } \\
\text { orous }\end{array}$ & $\mathrm{R}$ & $\begin{array}{c}\text { Least } \\
\text { concern }\end{array}$ \\
\hline $\begin{array}{c}13 \\
9\end{array}$ & $\begin{array}{c}\text { Passeriform } \\
\text { es }\end{array}$ & $\begin{array}{c}\text { Muscicapid } \\
\text { ae }\end{array}$ & Blue rockthrush & Monticola solitarius & $\begin{array}{c}\text { Insectiv } \\
\text { orous }\end{array}$ & $\mathrm{W}$ & $\begin{array}{c}\text { Least } \\
\text { concern }\end{array}$ \\
\hline $\begin{array}{c}14 \\
0\end{array}$ & $\begin{array}{c}\text { Passeriform } \\
\text { es }\end{array}$ & $\begin{array}{c}\text { Nectariniid } \\
\text { ae }\end{array}$ & Purple sunbird & Cinnyris asiaticus & $\begin{array}{c}\text { Nectivo } \\
\text { rous }\end{array}$ & $\mathrm{R}$ & $\begin{array}{c}\text { Least } \\
\text { concern }\end{array}$ \\
\hline $\begin{array}{c}14 \\
1\end{array}$ & $\begin{array}{c}\text { Passeriform } \\
\text { es }\end{array}$ & Oriolidae & Indian oriole & Oriolus oriolus & $\begin{array}{c}\text { Frugivo } \\
\text { rous }\end{array}$ & $\mathrm{S}$ & $\begin{array}{c}\text { Least } \\
\text { concern }\end{array}$ \\
\hline $\begin{array}{c}14 \\
2\end{array}$ & $\begin{array}{c}\text { Passeriform } \\
\text { es }\end{array}$ & Paridae & Great tit & Parus major & $\begin{array}{c}\text { Insectiv } \\
\text { orous }\end{array}$ & $\mathrm{R}$ & $\begin{array}{c}\text { Least } \\
\text { concern }\end{array}$ \\
\hline $\begin{array}{c}14 \\
3\end{array}$ & $\begin{array}{c}\text { Passeriform } \\
\text { es }\end{array}$ & Passeridae & $\begin{array}{l}\text { Chestnut } \\
\text { Shouldered } \\
\text { petronia }\end{array}$ & $\begin{array}{c}\text { Gymnoris } \\
\text { xanthocollis }\end{array}$ & $\begin{array}{c}\text { Granivo } \\
\text { rous }\end{array}$ & $\mathrm{R}$ & $\begin{array}{c}\text { Least } \\
\text { concern }\end{array}$ \\
\hline $\begin{array}{c}14 \\
4\end{array}$ & $\begin{array}{c}\text { Passeriform } \\
\text { es }\end{array}$ & Passeridae & House sparrow & Passer domesticus & $\begin{array}{c}\text { Granivo } \\
\text { rous }\end{array}$ & $\mathrm{R}$ & $\begin{array}{c}\text { Least } \\
\text { concern }\end{array}$ \\
\hline $\begin{array}{c}14 \\
5\end{array}$ & $\begin{array}{c}\text { Passeriform } \\
\text { es }\end{array}$ & $\begin{array}{c}\text { Phylloscop } \\
\text { idae }\end{array}$ & Common chiffchaff & $\begin{array}{c}\text { Phylloscopus } \\
\text { collybita }\end{array}$ & $\begin{array}{c}\text { Insectiv } \\
\text { orous }\end{array}$ & W & $\begin{array}{c}\text { Least } \\
\text { concern }\end{array}$ \\
\hline $\begin{array}{c}14 \\
6 \\
\end{array}$ & $\begin{array}{c}\text { Passeriform } \\
\text { es }\end{array}$ & $\begin{array}{c}\text { Phylloscop } \\
\text { idae }\end{array}$ & $\begin{array}{c}\text { Sulphur- bellied } \\
\text { warbler }\end{array}$ & $\begin{array}{l}\text { Phylloscopus } \\
\text { griseolus }\end{array}$ & $\begin{array}{c}\text { Insectiv } \\
\text { orous }\end{array}$ & $\mathrm{W}$ & $\begin{array}{c}\text { Least } \\
\text { concern }\end{array}$ \\
\hline $\begin{array}{c}14 \\
7\end{array}$ & $\begin{array}{c}\text { Passeriform } \\
\text { es }\end{array}$ & Ploceidae & Baya weaver & Ploceus philippinus & $\begin{array}{c}\text { Insectiv } \\
\text { orous }\end{array}$ & $\mathrm{R}$ & $\begin{array}{c}\text { Least } \\
\text { concern }\end{array}$ \\
\hline $\begin{array}{c}14 \\
8\end{array}$ & $\begin{array}{c}\text { Passeriform } \\
\text { es }\end{array}$ & $\begin{array}{c}\text { Pycnonotid } \\
\text { ae }\end{array}$ & White eared bulbul & Pycnonotus leucotis & $\begin{array}{c}\text { Frugivo } \\
\text { rous }\end{array}$ & $\mathrm{R}$ & $\begin{array}{c}\text { Least } \\
\text { concern }\end{array}$ \\
\hline $\begin{array}{c}14 \\
9\end{array}$ & $\begin{array}{c}\text { Passeriform } \\
\text { es }\end{array}$ & $\begin{array}{c}\text { Pycnonotid } \\
\text { ae }\end{array}$ & Red vented bulbul & Pycnonotus cafer & $\begin{array}{c}\text { Insectiv } \\
\text { orous }\end{array}$ & $\mathrm{R}$ & $\begin{array}{c}\text { Least } \\
\text { concern }\end{array}$ \\
\hline $\begin{array}{c}15 \\
0\end{array}$ & $\begin{array}{c}\text { Passeriform } \\
\text { es }\end{array}$ & $\begin{array}{c}\text { Rhipidurid } \\
\text { ae }\end{array}$ & $\begin{array}{l}\text { White browed } \\
\text { fantail }\end{array}$ & Rhipidura aureola & $\begin{array}{c}\text { Insectiv } \\
\text { orous }\end{array}$ & $\mathrm{R}$ & $\begin{array}{c}\text { Least } \\
\text { concern }\end{array}$ \\
\hline $\begin{array}{c}15 \\
1\end{array}$ & $\begin{array}{c}\text { Passeriform } \\
\text { es }\end{array}$ & $\begin{array}{c}\text { Stenostirid } \\
\text { ae }\end{array}$ & $\begin{array}{c}\text { Grey headed } \\
\text { cannery flycatcher }\end{array}$ & $\begin{array}{c}\text { Culicicapa } \\
\text { ceylonensis }\end{array}$ & $\begin{array}{c}\text { Insectiv } \\
\text { orous }\end{array}$ & W & $\begin{array}{c}\text { Least } \\
\text { concern }\end{array}$ \\
\hline $\begin{array}{c}15 \\
2\end{array}$ & $\begin{array}{c}\text { Passeriform } \\
\text { es }\end{array}$ & Sturnidae & Brahminy starling & Sturnia pagodarum & $\begin{array}{c}\text { Insectiv } \\
\text { orous }\end{array}$ & $\mathrm{R}$ & $\begin{array}{c}\text { Least } \\
\text { concern }\end{array}$ \\
\hline $\begin{array}{c}15 \\
3\end{array}$ & $\begin{array}{c}\text { Passeriform } \\
\text { es }\end{array}$ & Sturnidae & Asian pied starling & Gracupica contra & $\begin{array}{c}\text { Omnivo } \\
\text { rous }\end{array}$ & $\mathrm{R}$ & $\begin{array}{c}\text { Least } \\
\text { concern }\end{array}$ \\
\hline $\begin{array}{c}15 \\
4\end{array}$ & $\begin{array}{c}\text { Passeriform } \\
\text { es }\end{array}$ & Sturnidae & Common starling & Sturnus vulgaris & $\begin{array}{c}\text { Omnivo } \\
\text { rous }\end{array}$ & $\mathrm{W}$ & $\begin{array}{c}\text { Least } \\
\text { concern }\end{array}$ \\
\hline $\begin{array}{c}15 \\
5\end{array}$ & $\begin{array}{c}\text { Passeriform } \\
\text { es }\end{array}$ & Sturnidae & Rosy starling & Pastor roseus & $\begin{array}{c}\text { Omnivo } \\
\text { rous }\end{array}$ & W & $\begin{array}{c}\text { Least } \\
\text { concern }\end{array}$ \\
\hline $\begin{array}{c}15 \\
6\end{array}$ & $\begin{array}{c}\text { Passeriform } \\
\text { es }\end{array}$ & Sturnidae & Common myna & Acridotheres tristis & $\begin{array}{c}\text { Omnivo } \\
\text { rous }\end{array}$ & $\mathrm{R}$ & $\begin{array}{c}\text { Least } \\
\text { concern }\end{array}$ \\
\hline
\end{tabular}


Rounak Choudhary, Praveen Mathur, and Vivek Sharma

\begin{tabular}{|c|c|c|c|c|c|c|c|}
\hline $\begin{array}{c}15 \\
7\end{array}$ & $\begin{array}{c}\text { Passeriform } \\
\text { es }\end{array}$ & Sturnidae & Bank myna & $\begin{array}{l}\text { Acridotheres } \\
\text { ginginianus }\end{array}$ & $\begin{array}{l}\text { Omnivo } \\
\text { rous }\end{array}$ & $\mathrm{R}$ & $\begin{array}{c}\text { Least } \\
\text { concern }\end{array}$ \\
\hline $\begin{array}{c}15 \\
8\end{array}$ & $\begin{array}{c}\text { Passeriform } \\
\text { es } \\
\end{array}$ & Sylviidae & Lesser white throat & Sylvia curruca & $\begin{array}{c}\text { Insectiv } \\
\text { orous }\end{array}$ & W & $\begin{array}{c}\text { Least } \\
\text { concern }\end{array}$ \\
\hline $\begin{array}{c}15 \\
9 \\
\end{array}$ & $\begin{array}{c}\text { Passeriform } \\
\text { es } \\
\end{array}$ & Sylviidae & $\begin{array}{c}\text { Yellow Eyed } \\
\text { Babbler }\end{array}$ & Chrysomma sinense & $\begin{array}{c}\text { Insectiv } \\
\text { orous }\end{array}$ & $\mathrm{R}$ & $\begin{array}{c}\text { Least } \\
\text { concern }\end{array}$ \\
\hline $\begin{array}{c}16 \\
0\end{array}$ & $\begin{array}{c}\text { Passeriform } \\
\text { es }\end{array}$ & Vangidae & $\begin{array}{c}\text { Common } \\
\text { woodshrike }\end{array}$ & $\begin{array}{l}\text { Tephrodornis } \\
\text { pondicerianus }\end{array}$ & $\begin{array}{l}\text { Insectiv } \\
\text { orous }\end{array}$ & $S$ & $\begin{array}{c}\text { Least } \\
\text { concern }\end{array}$ \\
\hline $\begin{array}{c}16 \\
1\end{array}$ & $\begin{array}{c}\text { Passeriform } \\
\text { es }\end{array}$ & $\begin{array}{c}\text { Zosteropid } \\
\text { ae }\end{array}$ & Oriental white eye & $\begin{array}{c}\text { Zosterops } \\
\text { palpebrosus }\end{array}$ & $\begin{array}{c}\text { Omnivo } \\
\text { rous }\end{array}$ & $\mathrm{R}$ & $\begin{array}{c}\text { Least } \\
\text { concern }\end{array}$ \\
\hline $\begin{array}{c}16 \\
2 \\
\end{array}$ & $\begin{array}{c}\text { Pelecanifor } \\
\text { mes }\end{array}$ & Ardeidae & Cattle egret & Bubulcus ibis & $\begin{array}{l}\text { Insectiv } \\
\text { orous }\end{array}$ & $\mathrm{R}$ & $\begin{array}{c}\text { Least } \\
\text { concern }\end{array}$ \\
\hline $\begin{array}{c}16 \\
3\end{array}$ & $\begin{array}{c}\text { Pelecanifor } \\
\text { mes }\end{array}$ & Ardeidae & Little egret & Egretta garzetta & $\begin{array}{c}\text { Carnivo } \\
\text { rous }\end{array}$ & $\mathrm{R}$ & $\begin{array}{c}\text { Least } \\
\text { concern }\end{array}$ \\
\hline $\begin{array}{c}16 \\
4 \\
\end{array}$ & $\begin{array}{l}\text { Pelecanifor } \\
\text { mes }\end{array}$ & Ardeidae & Intermediate egret & Ardea intermedia & $\begin{array}{c}\text { Carnivo } \\
\text { rous }\end{array}$ & $\mathrm{R}$ & $\begin{array}{c}\text { Least } \\
\text { concern }\end{array}$ \\
\hline $\begin{array}{c}16 \\
5\end{array}$ & $\begin{array}{c}\text { Pelecanifor } \\
\text { mes }\end{array}$ & Ardeidae & Great egret & Ardea alba & $\begin{array}{l}\text { Carnivo } \\
\text { rous }\end{array}$ & $\mathrm{R}$ & $\begin{array}{c}\text { Least } \\
\text { concern }\end{array}$ \\
\hline $\begin{array}{c}16 \\
6 \\
\end{array}$ & $\begin{array}{l}\text { Pelecanifor } \\
\text { mes }\end{array}$ & Ardeidae & Indian pond heron & Ardeola grayii & $\begin{array}{l}\text { Carnivo } \\
\text { rous }\end{array}$ & $\mathrm{R}$ & $\begin{array}{c}\text { Least } \\
\text { concern }\end{array}$ \\
\hline $\begin{array}{c}16 \\
7\end{array}$ & $\begin{array}{l}\text { Pelecanifor } \\
\text { mes }\end{array}$ & Ardeidae & Purple Heron & Ardea purpurea & $\begin{array}{c}\text { Carnivo } \\
\text { rous }\end{array}$ & $\mathrm{R}$ & $\begin{array}{c}\text { Least } \\
\text { concern }\end{array}$ \\
\hline $\begin{array}{c}16 \\
8\end{array}$ & $\begin{array}{c}\text { Pelecanifor } \\
\text { mes }\end{array}$ & Ardeidae & Grey Heron & Ardea cinerea & $\begin{array}{c}\text { Carnivo } \\
\text { rous }\end{array}$ & $\mathrm{R}$ & $\begin{array}{c}\text { Least } \\
\text { concern }\end{array}$ \\
\hline $\begin{array}{c}16 \\
9\end{array}$ & $\begin{array}{l}\text { Pelecanifor } \\
\text { mes }\end{array}$ & Ardeidae & Little Green Heron & Butorides striatus & $\begin{array}{c}\text { Carnivo } \\
\text { rous }\end{array}$ & $\mathrm{R}$ & $\begin{array}{c}\text { Least } \\
\text { concern }\end{array}$ \\
\hline $\begin{array}{c}17 \\
0\end{array}$ & $\begin{array}{c}\text { Pelecanifor } \\
\text { mes }\end{array}$ & Ardeidae & $\begin{array}{l}\text { Black Crowned } \\
\text { night heron }\end{array}$ & $\begin{array}{l}\text { Nycticorax } \\
\text { nycticorax }\end{array}$ & $\begin{array}{l}\text { Carnivo } \\
\text { rous }\end{array}$ & $\mathrm{R}$ & $\begin{array}{c}\text { Least } \\
\text { concern }\end{array}$ \\
\hline $\begin{array}{c}17 \\
1\end{array}$ & $\begin{array}{c}\text { Pelecanifor } \\
\text { mes }\end{array}$ & $\begin{array}{c}\text { Threskiorn } \\
\text { ithidae }\end{array}$ & Black ibis & Pseudibis papillosa & $\begin{array}{l}\text { Carnivo } \\
\text { rous }\end{array}$ & $\mathrm{R}$ & $\begin{array}{c}\text { Least } \\
\text { concern }\end{array}$ \\
\hline $\begin{array}{c}17 \\
2\end{array}$ & $\begin{array}{c}\text { Pelecanifor } \\
\text { mes }\end{array}$ & $\begin{array}{c}\text { Threskiorn } \\
\text { ithidae }\end{array}$ & Glossy ibis & Plegadis falcinellus & $\begin{array}{c}\text { Carnivo } \\
\text { rous }\end{array}$ & $\mathrm{R}$ & $\begin{array}{c}\text { Least } \\
\text { concern }\end{array}$ \\
\hline $\begin{array}{c}17 \\
3\end{array}$ & $\begin{array}{l}\text { Pelecanifor } \\
\text { mes }\end{array}$ & $\begin{array}{c}\text { Threskiorn } \\
\text { ithidae }\end{array}$ & Oriental white ibis & $\begin{array}{c}\text { Threskiornis } \\
\text { melanocephalus }\end{array}$ & $\begin{array}{l}\text { Carnivo } \\
\text { rous }\end{array}$ & $\mathrm{R}$ & $\begin{array}{c}\text { Near } \\
\text { Threatene } \\
\mathrm{d}\end{array}$ \\
\hline $\begin{array}{c}17 \\
4\end{array}$ & $\begin{array}{l}\text { Pelecanifor } \\
\text { mes }\end{array}$ & $\begin{array}{c}\text { Threskiorn } \\
\text { ithidae }\end{array}$ & Eurasian Spoonbill & Platalea leucorodia & $\begin{array}{l}\text { Carnivo } \\
\text { rous }\end{array}$ & $\mathrm{R}$ & $\begin{array}{c}\text { Least } \\
\text { concern }\end{array}$ \\
\hline $\begin{array}{c}17 \\
5\end{array}$ & $\begin{array}{l}\text { Phoenicopte } \\
\text { riformes }\end{array}$ & $\begin{array}{c}\text { Phoenicopt } \\
\text { eridae }\end{array}$ & Lesser flamingo & $\begin{array}{l}\text { Phoenicopterus } \\
\text { minor }\end{array}$ & $\begin{array}{l}\text { Omnivo } \\
\text { rous }\end{array}$ & W & $\begin{array}{c}\text { Near } \\
\text { Threatene } \\
\text { d }\end{array}$ \\
\hline $\begin{array}{c}17 \\
6\end{array}$ & $\begin{array}{l}\text { Phoenicopte } \\
\text { riformes }\end{array}$ & $\begin{array}{c}\text { Phoenicopt } \\
\text { eridae }\end{array}$ & Greater flamingo & $\begin{array}{c}\text { Phoenicopterus } \\
\text { ruber }\end{array}$ & $\begin{array}{l}\text { Omnivo } \\
\text { rous }\end{array}$ & W & $\begin{array}{c}\text { Least } \\
\text { concern }\end{array}$ \\
\hline $\begin{array}{c}17 \\
7 \\
\end{array}$ & Piciformes & $\begin{array}{l}\text { Megalaimi } \\
\text { dae }\end{array}$ & $\begin{array}{c}\text { Coppersmith } \\
\text { barbet }\end{array}$ & $\begin{array}{c}\text { Psilopogon } \\
\text { haemacephalus }\end{array}$ & $\begin{array}{l}\text { Frugivo } \\
\text { rous }\end{array}$ & $\mathrm{R}$ & $\begin{array}{c}\text { Least } \\
\text { concern }\end{array}$ \\
\hline $\begin{array}{c}17 \\
8\end{array}$ & Piciformes & Picidae & Eurasian wryneck & Jynx torquilla & $\begin{array}{l}\text { Insectiv } \\
\text { orous }\end{array}$ & W & $\begin{array}{c}\text { Least } \\
\text { concern }\end{array}$ \\
\hline $\begin{array}{c}17 \\
9\end{array}$ & Piciformes & Picidae & $\begin{array}{c}\text { Yellow crowned } \\
\text { woodpecker }\end{array}$ & $\begin{array}{c}\text { Leiopicus } \\
\text { mahrattensis }\end{array}$ & $\begin{array}{l}\text { Frugivo } \\
\text { rous }\end{array}$ & $\mathrm{R}$ & $\begin{array}{c}\text { Least } \\
\text { concern }\end{array}$ \\
\hline $\begin{array}{c}18 \\
0\end{array}$ & Piciformes & Picidae & $\begin{array}{l}\text { Black rumped } \\
\text { flamback }\end{array}$ & $\begin{array}{c}\text { Dinopium } \\
\text { benghalense }\end{array}$ & $\begin{array}{l}\text { Insectiv } \\
\text { orous }\end{array}$ & $\mathrm{R}$ & $\begin{array}{c}\text { Least } \\
\text { concern }\end{array}$ \\
\hline $\begin{array}{c}18 \\
1\end{array}$ & $\begin{array}{l}\text { Podicipedifo } \\
\text { rmes }\end{array}$ & $\begin{array}{c}\text { Podicipedi } \\
\text { dae }\end{array}$ & Little grebe & $\begin{array}{l}\text { Tachybaptus } \\
\text { ruficollis }\end{array}$ & $\begin{array}{l}\text { Carnivo } \\
\text { rous }\end{array}$ & $\mathrm{R}$ & $\begin{array}{c}\text { Least } \\
\text { concern }\end{array}$ \\
\hline
\end{tabular}


Avifaunal Composition of Various Microhabitats of Southern Nagaur (Parbatsar, Kuchaman, Nawa and Makrana), Rajasthan

\begin{tabular}{|c|c|c|c|c|c|c|c|}
\hline $\begin{array}{c}18 \\
2\end{array}$ & $\begin{array}{c}\text { Psittaciform } \\
\text { es }\end{array}$ & $\begin{array}{c}\text { Psittaculid } \\
\text { ae }\end{array}$ & $\begin{array}{c}\text { Rose ringed } \\
\text { parakeet }\end{array}$ & Psittacula krameri & $\begin{array}{l}\text { Frugivo } \\
\text { rous }\end{array}$ & $\mathrm{R}$ & $\begin{array}{c}\text { Least } \\
\text { concern }\end{array}$ \\
\hline $\begin{array}{c}18 \\
3\end{array}$ & $\begin{array}{c}\text { Psittaciform } \\
\text { es }\end{array}$ & $\begin{array}{c}\text { Psittaculid } \\
\text { ae }\end{array}$ & $\begin{array}{l}\text { Plum headed } \\
\text { parakeet }\end{array}$ & $\begin{array}{c}\text { Psittacula } \\
\text { cyanocephala }\end{array}$ & $\begin{array}{l}\text { Frugivo } \\
\text { rous }\end{array}$ & $\mathrm{R}$ & $\begin{array}{c}\text { Least } \\
\text { concern }\end{array}$ \\
\hline $\begin{array}{c}18 \\
4\end{array}$ & $\begin{array}{c}\text { Psittaciform } \\
\text { es }\end{array}$ & $\begin{array}{c}\text { Psittaculid } \\
\text { ae }\end{array}$ & $\begin{array}{c}\text { Alexandrine } \\
\text { parakeet }\end{array}$ & Psittacula eupatria & $\begin{array}{l}\text { Frugivo } \\
\text { rous }\end{array}$ & $\mathrm{R}$ & $\begin{array}{c}\text { Near } \\
\text { Threatene } \\
\text { d }\end{array}$ \\
\hline $\begin{array}{c}18 \\
5\end{array}$ & $\begin{array}{c}\text { Pterocliform } \\
\text { es }\end{array}$ & Pteroclidae & $\begin{array}{c}\text { Chestnut bellied } \\
\text { sand grouse }\end{array}$ & Pterocles exustus & $\begin{array}{l}\text { Granivo } \\
\text { rous }\end{array}$ & $\mathrm{R}$ & $\begin{array}{c}\text { Least } \\
\text { concern }\end{array}$ \\
\hline $\begin{array}{c}18 \\
6\end{array}$ & Strigiformes & Strigidae & Indian Eagle Owl & Bubo bengalensis & $\begin{array}{l}\text { Carnivo } \\
\text { rous }\end{array}$ & $\mathrm{W}$ & $\begin{array}{c}\text { Least } \\
\text { concern }\end{array}$ \\
\hline $\begin{array}{c}18 \\
7\end{array}$ & Strigiformes & Strigidae & Spotted owlet & Athene brama & $\begin{array}{l}\text { Carnivo } \\
\text { rous }\end{array}$ & $\mathrm{R}$ & $\begin{array}{c}\text { Least } \\
\text { concern }\end{array}$ \\
\hline $\begin{array}{c}18 \\
8 \\
\end{array}$ & Strigiformes & Tytonidae & Barn owl & Tyto alba & $\begin{array}{l}\text { Carnivo } \\
\text { rous }\end{array}$ & $\mathrm{R}$ & $\begin{array}{c}\text { Least } \\
\text { concern }\end{array}$ \\
\hline $\begin{array}{c}18 \\
9\end{array}$ & Suliformes & $\begin{array}{l}\text { Phalacroco } \\
\text { racidae }\end{array}$ & Great cormorant & $\begin{array}{c}\text { Phalacrocorax } \\
\text { carbo }\end{array}$ & $\begin{array}{l}\text { Carnivo } \\
\text { rous }\end{array}$ & W & $\begin{array}{c}\text { Least } \\
\text { concern }\end{array}$ \\
\hline $\begin{array}{c}19 \\
0\end{array}$ & Suliformes & $\begin{array}{c}\text { Phalacroco } \\
\text { racidae }\end{array}$ & Indian shag & $\begin{array}{l}\text { Phalacrocorax } \\
\text { fuscicollis }\end{array}$ & $\begin{array}{l}\text { Carnivo } \\
\text { rous }\end{array}$ & $\mathrm{W}$ & $\begin{array}{c}\text { Least } \\
\text { concern }\end{array}$ \\
\hline $\begin{array}{c}19 \\
1\end{array}$ & Suliformes & $\begin{array}{l}\text { Phalacroco } \\
\text { racidae }\end{array}$ & Little cormorant & $\begin{array}{c}\text { Phalacrocorax } \\
\text { niger }\end{array}$ & $\begin{array}{l}\text { Carnivo } \\
\text { rous }\end{array}$ & $\mathrm{R}$ & $\begin{array}{c}\text { Least } \\
\text { concern }\end{array}$ \\
\hline
\end{tabular}

Table 2: Order wise Occurrence of observed species

\begin{tabular}{|c|c|c|}
\hline S. No. & Order & No. of Species \\
\hline 1 & Accipitriformes & 12 \\
\hline 2 & Anseriformes & 14 \\
\hline 3 & Apodiformes & 1 \\
\hline 4 & Bucerotiformes & 2 \\
\hline 5 & Charadriiformes & 28 \\
\hline 6 & Ciconiiformes & 3 \\
\hline 7 & Columbiformes & 6 \\
\hline 8 & Coraciiformes & 8 \\
\hline 9 & Cuculiformes & 4 \\
\hline 10 & Falconiformes & 1 \\
\hline 11 & Galliformes & 5 \\
\hline 12 & Gruiformes & 6 \\
\hline 13 & Passeriformes & 71 \\
\hline 14 & Pelecaniformes & 13 \\
\hline 15 & Phoenicopteriformes & 2 \\
\hline 16 & Piciformes & 4 \\
\hline 17 & Podicipediformes & 1 \\
\hline 18 & Psittaciformes & 3 \\
\hline 19 & Pterocliformes & 1 \\
\hline 20 & Strigiformes & 3 \\
\hline 21 & Suliformes & 3 \\
\hline
\end{tabular}


Rounak Choudhary, Praveen Mathur, and Vivek Sharma

Table 3: Family wise Occurrence of observed species along with their RDi

\begin{tabular}{|c|c|c|c|}
\hline S. No. & Family & No. of Species & RDi\% \\
\hline 1 & Accipitridae & 11 & 5.76 \\
\hline 2 & Pandionidae & 1 & 0.52 \\
\hline 3 & Anatidae & 14 & 7.33 \\
\hline 4 & Apodidae & 1 & 0.52 \\
\hline 5 & Upupidae & 1 & 0.52 \\
\hline 6 & Bucerotidae & 1 & 0.52 \\
\hline 7 & Burhinidae & 2 & 1.05 \\
\hline 8 & Charadriidae & 4 & 2.09 \\
\hline 9 & Glareolidae & 1 & 0.52 \\
\hline 10 & Laridae & 6 & 3.14 \\
\hline 11 & Recurvirostridae & 2 & 1.05 \\
\hline 12 & Rostratulidae & 1 & 0.52 \\
\hline 13 & Scolopacidae & 11 & 5.76 \\
\hline 14 & Turnicidae & 1 & 0.52 \\
\hline 15 & Ciconiidae & 3 & 1.57 \\
\hline 16 & Columbidae & 6 & 3.14 \\
\hline 17 & Alcedinidae & 3 & 1.57 \\
\hline 18 & Coraciidae & 2 & 1.05 \\
\hline 19 & Meropidae & 3 & 1.57 \\
\hline 20 & Cuculidae & 4 & 2.09 \\
\hline 21 & Falconidae & 1 & 0.52 \\
\hline 22 & Phasianidae & 5 & 2.62 \\
\hline 23 & Gruidae & 2 & 1.05 \\
\hline 24 & Rallidae & 4 & 2.09 \\
\hline 25 & Alaudidae & 6 & 3.14 \\
\hline 26 & Campephagidae & 2 & 1.05 \\
\hline 27 & Certhiidae & 1 & 0.52 \\
\hline 28 & Cisticolidae & 6 & 3.14 \\
\hline 29 & Corvidae & 2 & 1.05 \\
\hline 30 & Dicruridae & 2 & 1.05 \\
\hline 31 & Emberizidae & 2 & 1.05 \\
\hline 32 & Estrildidae & 1 & 0.52 \\
\hline 33 & Hirundinidae & 4 & 2.09 \\
\hline 34 & Laniidae & 3 & 1.57 \\
\hline 35 & Leiothrichidae & 3 & 1.57 \\
\hline 36 & Motacillidae & 5 & 2.62 \\
\hline 37 & Muscicapidae & 12 & 6.28 \\
\hline 38 & Nectariniidae & 1 & 0.52 \\
\hline 39 & Oriolidae & 1 & 0.52 \\
\hline 40 & Paridae & 1 & 0.52 \\
\hline 41 & Passeridae & 2 & 1.05 \\
\hline 42 & Phylloscopidae & 2 & 1.05 \\
\hline 43 & Ploceidae & 1 & 0.52 \\
\hline 44 & Pycnonotidae & 2 & 1.05 \\
\hline 45 & Rhipiduridae & 1 & 0.52 \\
\hline 46 & Stenostiridae & 1 & 0.52 \\
\hline 47 & Sturnidae & 6 & 3.14 \\
\hline 48 & Sylviidae & 2 & 1.05 \\
\hline 49 & Vangidae & 1 & 0.52 \\
\hline
\end{tabular}


Avifaunal Composition of Various Microhabitats of Southern Nagaur (Parbatsar, Kuchaman, Nawa and Makrana), Rajasthan

\begin{tabular}{|c|c|c|c|}
\hline 50 & Zosteropidae & 1 & 0.52 \\
\hline 51 & Ardeidae & 9 & 4.71 \\
\hline 52 & Threskiornithidae & 4 & 2.09 \\
\hline 53 & Phoenicopteridae & 2 & 1.05 \\
\hline 54 & Megalaimidae & 1 & 0.52 \\
\hline 55 & Picidae & 3 & 1.57 \\
\hline 56 & Podicipedidae & 1 & 0.52 \\
\hline 57 & Psittaculidae & 3 & 1.57 \\
\hline 58 & Pteroclidae & 1 & 0.52 \\
\hline 59 & Strigidae & 2 & 1.05 \\
\hline 60 & Tytonidae & 1 & 0.52 \\
\hline \multirow[t]{2}{*}{61} & Phalacrocoracidae & 3 & 1.57 \\
\hline & Total & 191 & 100.00 \\
\hline
\end{tabular}

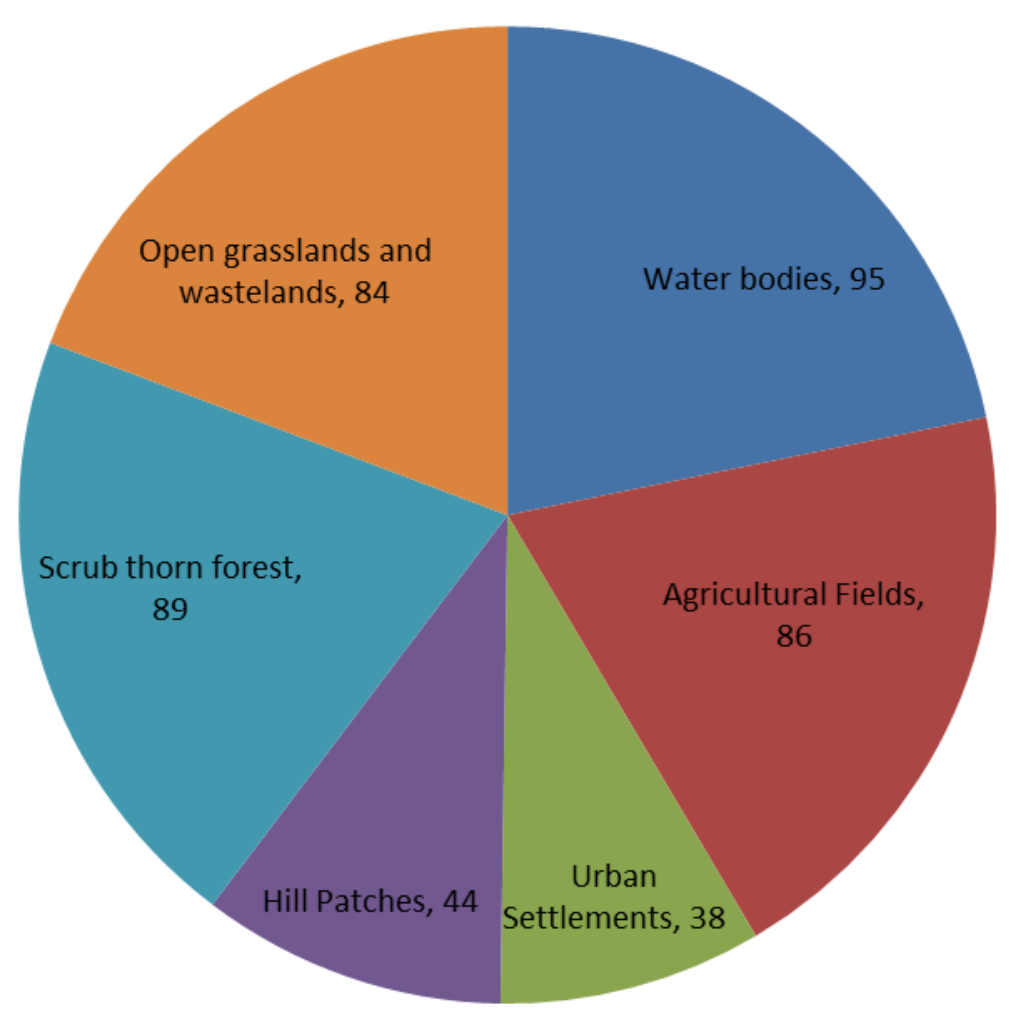

Figure 2: Observed Species in Different Classified Microhabitats

Table 4: Feeding Guild and their Percentage in Overall Population of Observed Species at Study Area

\begin{tabular}{|c|c|c|c|}
\hline S. No. & Feeding Guild & No. of Species & Percentage \\
\hline 1 & Carnivorous & 45 & $23.5 \%$ \\
\hline 2 & Frugivorous & 10 & $5.2 \%$ \\
\hline 3 & Granivorous & 24 & $12.5 \%$ \\
\hline 4 & Herbivorous & 11 & $5.7 \%$ \\
\hline 5 & Insectivorous & 77 & $40.3 \%$ \\
\hline 6 & Nectivorous & 1 & $0.5 \%$ \\
\hline 7 & Omnivorous & 23 & $12 \%$ \\
\hline
\end{tabular}




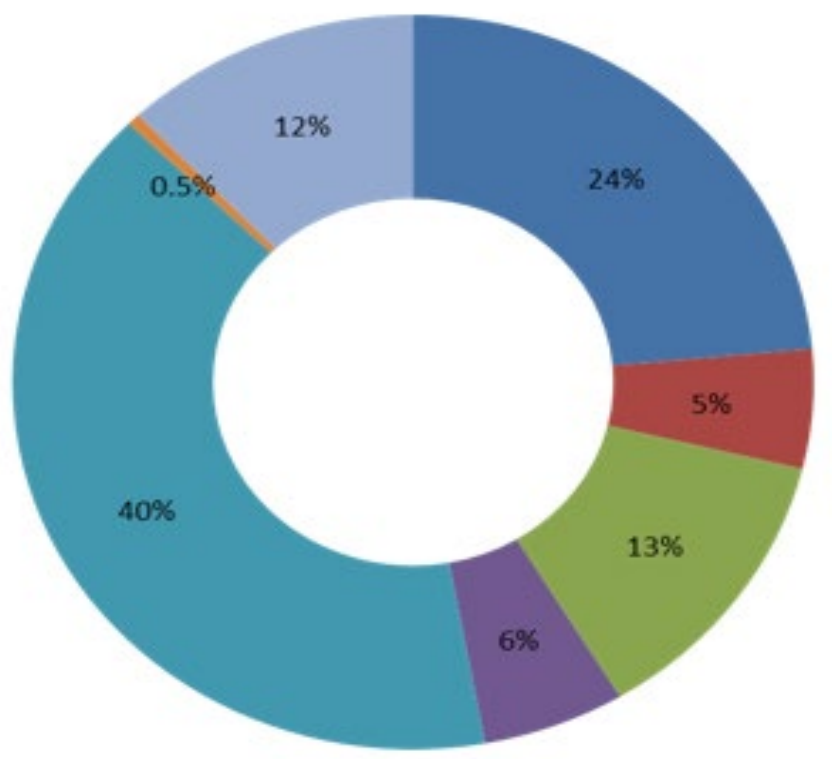

$$
\begin{aligned}
& \text { mCarnivorous }=\text { Frugivorous }=\text { Granivorous }=\text { Herbivorous } \\
& \text { minsectivorous } \equiv \text { Nectivorous }=\text { Omnivorous }
\end{aligned}
$$

Figure 3: Feeding Guild of species in study area

Table 5: Migratory Status of Observed Species at Study Area

\begin{tabular}{|c|c|c|}
\hline S. No. & Migratory Status & No. of Species \\
\hline 1 & Residential & 119 \\
\hline 2 & Summer Migratory & 5 \\
\hline 3 & Winter Migratory & 67 \\
\hline
\end{tabular}

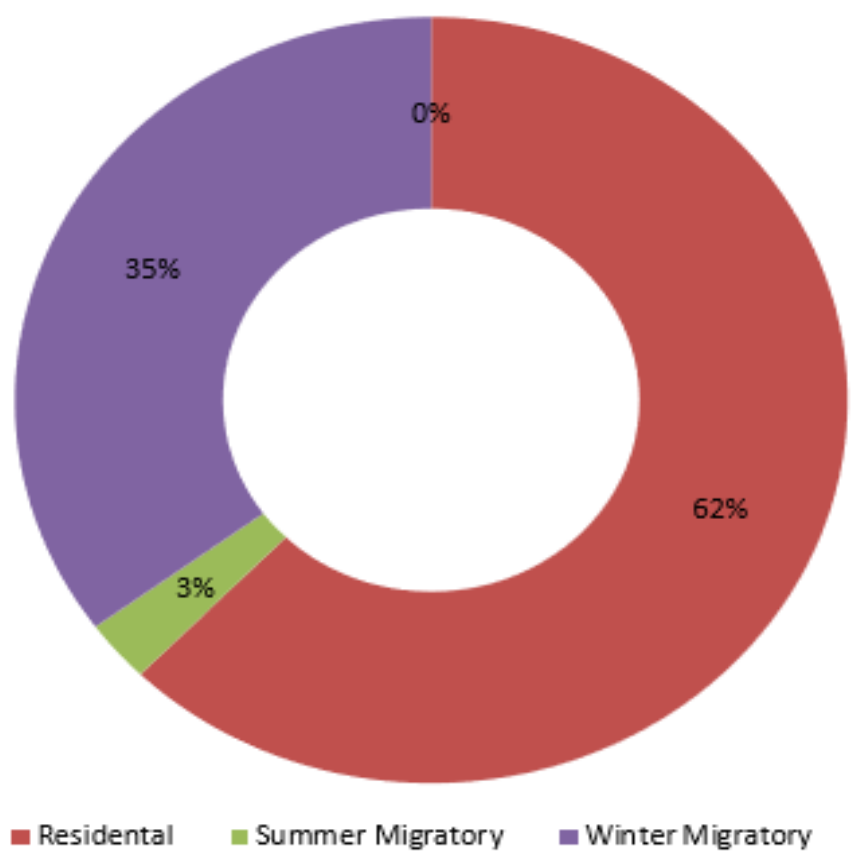

Figure 4: Migratory status of species in study area 
Table 6: IUCN Status of Observed Species at Study Area

\begin{tabular}{|c|c|c|}
\hline S. No. & IUCN Status & No. of Species \\
\hline 1 & Endangered & 2 \\
\hline 2 & Least concern & 178 \\
\hline 3 & Near Threatened & 8 \\
\hline 4 & Vulnerable & 3 \\
\hline
\end{tabular}

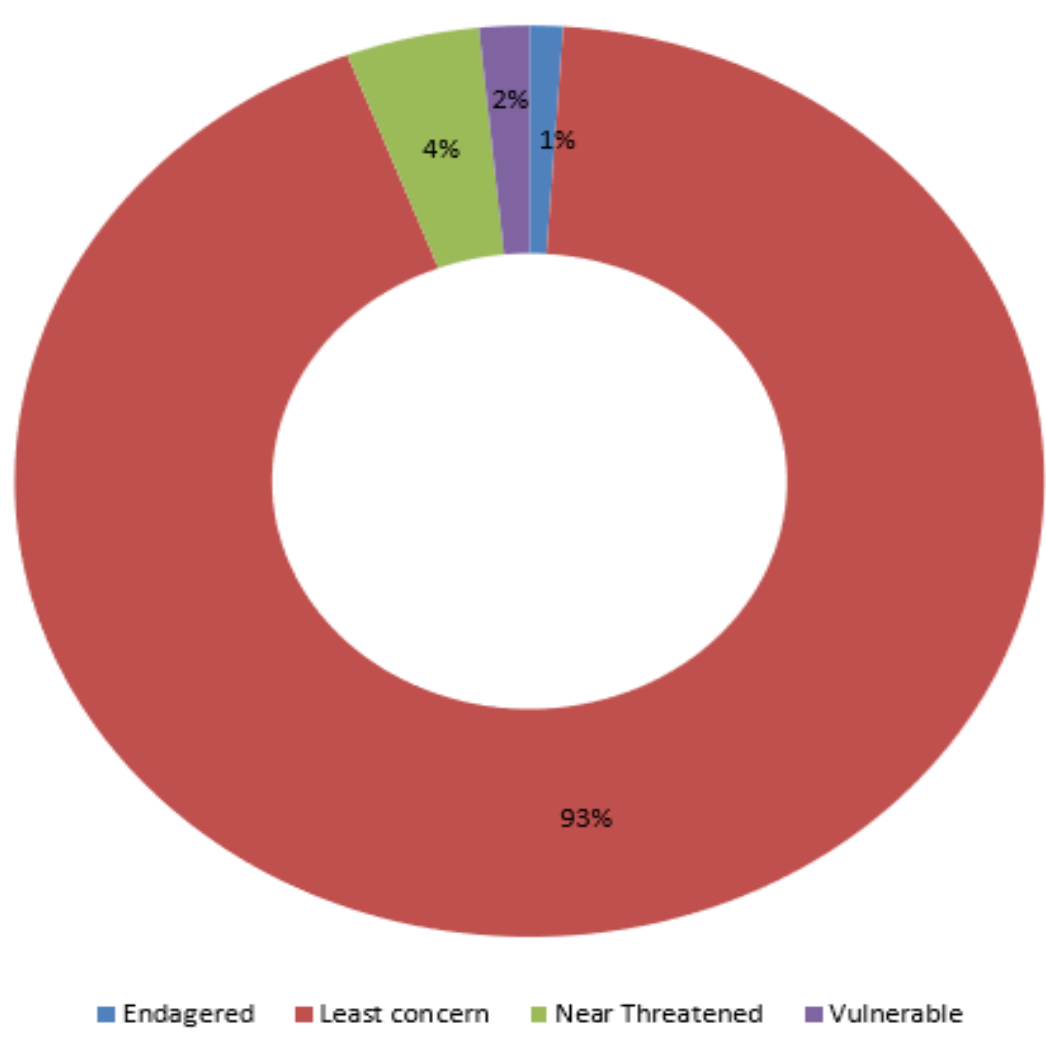

Figure 5: IUCN status of species in study area

\section{CONCLUSIONS AND RECOMMENDATIONS}

A total of 191 Avifaunal species belonging to 21 Orders and 61 Families were recorded from study area. The dominance among families was exhibited by family Anatidae (14 species with 7.33\%, RDi) followed by Muscicapidae (12 species with 6.28\% RDi), Accipitridae (11 species with 5.76\% RDi) and Scolopacide (11 species with 5.76\% RDi) respectively. Seven Feeding Guilds were identified during the study i.e., Insectivore (77 species) followed by Carnivores ( 45 species), Granivores ( 24 species), Omnivores (23 species), Herbivores (11 species), Frugivores (10 species) and Nectivores (1 species) based on the food preferences of the Avifauna. Out of 191 species recorded 72 species were Migratory (5 Summer Migratory \& 67 Winter Migratory).

178 Species listed as Least Concern Species, 8 Species listed as Near Threatened species, 3 Species listed as Vulnerable and 2 Species were listed as Endangered under the IUCN Categories.

Most of birds inhabiting these areas are vulnerable to habitat degradation due to flaws in existing legal frameworks. In addition, community knowledge enhancements also should be strengthened for a sustainable conservation of bird species while maintaining their ecological interactions. Meanwhile Nagaur district act as a transition zone between Arid and Semi-arid habitats of Rajasthan but unfortunately still does not have any Important Bird \& Biodiversity Area (IBA), it is strongly recommended to propose an Important Bird \& Biodiversity Area (IBA). Simultaneously, further long-term studies are recommended that covering migratory seasons for the bird species along with and documentation and population assessment of other components of biodiversity that ultimately enhance the knowledge about the diversity and its importance to the people at the study area. 


\section{SOURCES OF FUNDING}

This research received no specific grant from any funding agency in the public, commercial, or not-for-profit sectors.

\section{CONFLICT OF INTEREST}

The author have declared that no competing interests exist.

\section{ACKNOWLEDGMENT}

None.

\section{REFERENCES}

[1] Able, K.P. (1995), Orientation and navigation: a perspective on fifty years of research Condor 97:592-604.

[2] Ali, S. (1996), The Book of Indian Birds. Bombay Natural History Society. Mumbai. pp. 1-354.

[3] BNHS (2011b), ENVIS Centre for avian ecology. Available at http://www.bnhsenvis.nic.in Raiasthan. Accessed on 25th September, 2011.

[4] Cody, M.L. (1978), Habitat selection and interspecific territoriality among the sylviid warblers of England and Sweden. Ecological Monograph, 48(4): 351-396.

[5] Grimmet, R., Inskipp, C. \& Inskipp, T. (1998), Birds of the Indian subcontinent. Oxford University Press, Delhi, 1-888.

[6] International Union for Conservation of Nature (2020), Guidelines for Using the IUCN Red List Categories and Criteria (PDF)

[7] Islam, M.Z. \& Rahmani, A.R. (2004), Important Bird Areas in India: Priority sites for conservation. Indian Bird Conservation Network, Bombay Natural History Society and BirdLife International (UK), pp 1133.

[8] Simberioff, D. \& Dayan, T. (1991), The guild concept and the structure of ecological communities. Ann. Rev. Ecol. Syst. 22: 115-143.

[9] Sorensen, T. (1948), A method of establishing groups of equal amplitude in plant sociology based on similarity of species content and its application to analyses of the vegetation on Danish commons. Kongelige Danske Videnskabernes Selskab 5: 1-34

[10] Urfi, A., Sen, M., Kalam, A. \& Meganathan, T. (2005), Counting birds in India: Methodologies and trends. Current Science, 89.

[11] Wiens, J.A. (1989), Ecology of bird communities. Cambridge University Press, Cambride, Vol. $1 \& 2$. 